\title{
Relations between Hall Plates with Complementary Contact Geometries
}

\author{
Udo Ausserlechner \\ Department Sense and Control, Infineon Technologies AG, Villach, Austria \\ Email: udo.ausserlechner@infineon.com
}

How to cite this paper: Ausserlechner, U. (2019) Relations between Hall Plates with Complementary Contact Geometries. Journal of Applied Mathematics and Physics, 7, 2836-2867.

https://doi.org/10.4236/jamp.2019.711195

Received: October 19, 2019

Accepted: November 17, 2019

Published: November 20, 2019

Copyright (c) 2019 by author(s) and Scientific Research Publishing Inc. This work is licensed under the Creative Commons Attribution International License (CC BY 4.0).

http://creativecommons.org/licenses/by/4.0/

\section{(c) (7) Open Access}

\begin{abstract}
Singly connected Hall plates with $N$ peripheral contacts can be mapped onto the upper half of the $z$-plane by a conformal transformation. Recently, Homentcovschi and Bercia derived the General Formula for the electric field in this region. We present an alternative intuitive derivation based on conformal mapping arguments. Then we apply the General Formula to complementary Hall plates, where contacts and insulating boundaries are swapped. The resistance matrix of the complementary device at reverse magnetic field is expressed in terms of the conductance matrix of the original device at non-reverse magnetic field. These findings are used to prove several symmetry properties of Hall plates and their complementary counterparts at arbitrary magnetic field.
\end{abstract}

\section{Keywords}

Complementary Hall Plate, Conformal Mapping, Hall Plate,

Reverse Magnetic Field on Complementary Device, Resistance Matrix,

Reverse Magnetic Field Reciprocity, Stream Function, Calculus of Residues

\section{Introduction}

The purpose of this work is to derive relations between Hall plates and their complementary counter-parts. A Hall plate is assumed to be a plane conductive region with thickness much smaller than its lateral dimensions. We discuss only singly-connected Hall plates without holes. An arbitrary number of at least two extended contacts are on the perimeter. Between neighboring contacts there is a unitary piece of insulating boundary. Thus the number of contacts equals the number of unitary pieces of insulating boundary. The complementary Hall plate comprises the very same conductive region, but contacts and insulating boundaries on the perimeter are swapped. A complementary Hall plate has the same 
number of contacts as the original Hall plate. If the contacts of the original Hall plate are small, the complementary Hall plate has large contacts. Therefore a high impedance original device will generally have a low impedance complementary device. The first question is if there is a more precise quantitative relation between both devices. The ultimate question is if we can obtain all electrical parameters of the complementary device from the parameters of the original Hall plate.

Several properties of complementary Hall plates at zero or weak applied magnetic field have already been studied in the past. We found the following ones.

Plain distributed resistive structures with complementary peripheral electrode geometries were studied at zero magnetic field in [1]. The number of extended electrodes was $N>1$. The authors called the complementary device the dual device. They defined a first resistance between two non-neighboring contacts in the original device and a second resistance between two terminals in the dual device (see their Figure 2). The first terminal was connected to all contacts of the dual device, which are left of the two non-neighboring contacts in the original device. The second terminal was connected to all contacts of the dual device, which are right of the two non-neighboring contacts in the original device (see Figure 5 in this work). Finally, the product of these two resistances equals the square of the sheet resistance $R_{\text {sheet }}=\rho / t_{H}$, with the specific volume resistivity $\rho$ and the thickness of the Hall plate $t_{H}$. In [1] the authors also gave a general relation between indefinite impedance matrix $Z_{(N \times N)}$ and indefinite admittance matrix $\boldsymbol{Y}_{(N \times N)}$ of original and dual devices at zero magnetic field. Thereby, the impedance matrix of the complementary Hall plate is fully given by the admittance matrix of the original Hall plate. The goal of the current paper is to find an analogue relation that also holds in the presence of applied magnetic field of arbitrary strength. The arguments in [1] were based on the idea that potential and stream function are swapped if contacts and insulation boundaries are swapped. This naturally raises the question of what happens in cases where no stream function exists [2]. Further insight into the symmetry of such devices is given in [3].

Van-der-Pauw measurement on Hall plates with $90^{\circ}$ symmetry at zero magnetic field was discussed in [4]. There the authors focused on Hall plates with point-sized contacts and on their complementary counterparts of large contacts with no insulating boundaries in-between. Both cases can be readily computed in closed form. Then the authors found a smart power law, which interpolates the sheet resistance up to an astonishing accuracy of $\pm 0.02 \%$ for all contact sizes ((7) in [4], also (24) in [5]).

In [6] Van-der-Pauw measurement on rectangular Hall plates was studied at zero magnetic field. The devices had four extended contacts with two orthogonal symmetry axes. There the original device was labeled with "even symmetry" and the complementary device with "odd symmetry". If opposite contacts are shorted each device has only two terminals with the so-called cross resistance between them. It was found that the sheet resistance at zero magnetic field is twice the square-root of the product of cross resistances of original device and comple- 
mentary device (see (13a) in [6]).

In [7] plane singly-connected Hall plates with four peripheral contacts and equal input and output resistances were considered. If magnetic field is impressed on such a device, it has the same output voltage as its complementary device, provided both are supplied by the same voltage source (see Figure 7 in this work). This was conjectured in [8] and proven in [7] and [9] for weak applied magnetic field (see (50) in [8], see Section 4, Appendix B, and Figure 8, all in [7]). Numerical inspection suggests that this also holds for strong magnetic field, but a rigorous proof has not been given so far. In [8] it was also implicitly mentioned that the product of input resistances of original and complementary Hall plates of that particular symmetry (i.e., input resistance equals output resistance) at zero magnetic field equals twice the square of the sheet resistance (see the paragraph after (50) in [8]).

Complementary Hall plates with three extended contacts on the perimeter were studied in [10]. If such a device has single mirror symmetry, also its complementary device has single mirror symmetry. Then-analogous to above-the change of the potentials on the output contacts due to reversal of magnetic field polarity are identical in both original and complementary devices, if both devices are supplied with the same supply voltage on the other two contacts, and if the magnetic field is weak (see also Figure 6 in this work). This property also means that the ratio of Hall output signal over thermal noise under the constraint of fixed supply voltage and fixed input resistance is the same in the original Hall plate and in the complementary Hall plate [10] [11].

In Section 2 we reconsider the General Formula of [12] for the electric field in the upper half of the $z$-plane with $N$ contacts on the real axis. Thereby, we present a different derivation than the one given in [12]. This new approach shows how the stagnation points are linked to the electric field in the Hall plate. From this result we derive the resistance matrix of a general device at arbitrary magnetic field in Section 3. This is similar to [12]. In Section 4 we link the resistance matrices of original device and complementary device at reverse magnetic field. We call this the Reverse Magnetic Field on Complementary Device theorem (RMFoCD) - it is the core result of this work. In Section 5 we generalize the impedance relation in [1] for Hall plates at arbitrary magnetic field. In Sections 6 and 7 we prove that voltage supplied complementary Hall plates with three and four contacts have the same magnetic field sensitivities as the original Hall plates, if they are sufficiently symmetric. In Appendix A we evaluate an integral with the calculus of residues. Appendix $B$ defines several matrices and matrix manipulations used throughout this work. Appendix $C$ gives a numerical example, where the theory is compared to results of finite element simulations.

\section{The General Formula for the Electric Field}

Recently Homentcovschi and Bercia found a closed form analytical solution for the electric field in the upper half plane with an arbitrary number of extended contacts on the real axis [12]. The geometry of the problem is shown in Figure 1. 
The Hall plate has $N$ contacts with end points $a_{n}, b_{n}$ with the sequential order $b_{1}<a_{2}<b_{2}<a_{3}<\cdots<a_{N}<b_{N}<a_{N+1}=a_{1}$. Thus, contacts 2 to $N$ are lined up from left to right and contact 1 is at infinity. According to [12] the complex electric field $E(z)$ in the point $z=x+i y$ with $y \geq 0$ ( $i$ being the imaginary unit) is given by

$$
E(z)=E_{x}-i E_{y}=\frac{i}{L(z)} \sum_{n=1}^{N} c_{n} z^{n-1} \text { with } c_{n} \in \mathrm{R}
$$

with

$$
\begin{gathered}
L(z)=\prod_{n=1}^{N}\left(z-a_{n}\right)^{1-\gamma}\left(z-b_{n}\right)^{\gamma} \\
\gamma=\frac{1}{2}+\frac{\theta_{H}}{\pi} \text { with } 0<\gamma<1
\end{gathered}
$$

In (1c) $\theta_{H}$ is the Hall angle defined by $\tan \left(\theta_{H}\right)=\mu_{H} B_{\perp}$. We define the Hall mobility $\mu_{H}>0$ for negative charge carriers and $\mu_{H}<0$ for positive ones. This is opposite to the definition in [12] and therefore a different sign shows up in (1c). The applied magnetic flux density $B_{\perp}$ perpendicular to the Hall plate is counted positive, when it points out of the drawing plane.

In [12] Homentcovschi and Bercia derived their General Formula (1a) in a formal, "mathematical" way. Here we present a more intuitive derivation, which also sheds light on the physical meaning of the coefficients $C_{n}$. Figure 2 shows a Hall plate in the $w$-plane with the shape of a skewed parallelogram according to the method of Wick [13]. It is obtained from the Hall plate in Figure 1 by conformal mapping (after Schwartz-Christoffel). The skew angle is identical to the Hall angle. If the top and bottom contacts are supplied with electric energy, the current streamlines will be homogeneous and parallel to the left and right edges of the parallelogram, and the equipotential lines will be horizontal and evenly spaced. Thus the electric field will be vertical and homogeneous. As for any Hall plate the angle between the current density $S(w)$ and the electric field $E(w)$ is identical to the Hall angle for all points in the Hall plate:

$$
S(w)=S_{u}-i S_{v}=\frac{1}{\rho} \cos \left(\theta_{H}\right) \exp \left(-i \theta_{H}\right) E(w)
$$

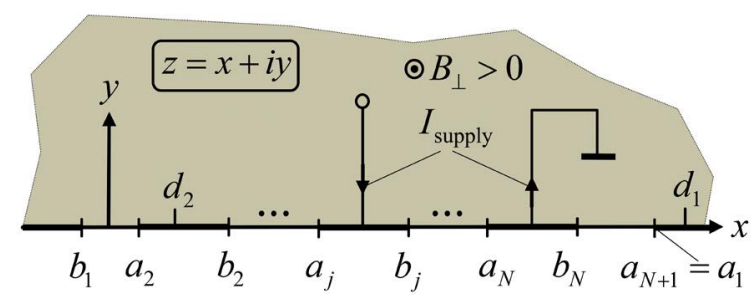

Figure 1. Hall plate in the infinite upper half of the $z$-plane with $N$ peripheral contacts on the real axis. The magnetic field points out of the drawing plane. Current is injected at contact $j$ and extracted at ground contact $N$. Contact 1 is at infinity. Points $d_{n}$ are stagnation points on all $N-2$ output contacts (supply contacts $j$ and $N$ have no stagnation points). 


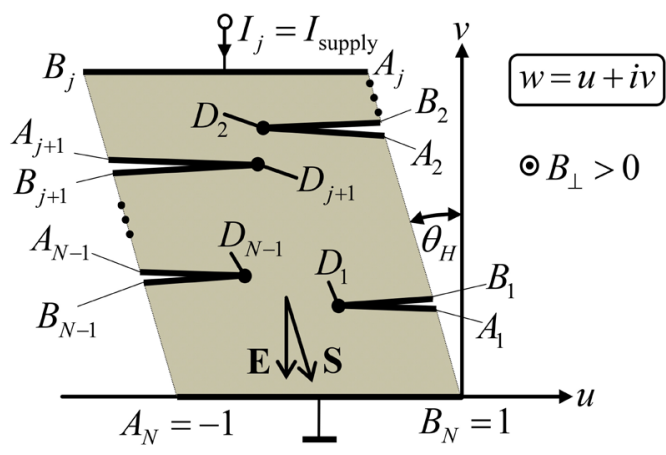

Figure 2. Hall plate as a skewed parallelogram in the $w$-plane with $N$ peripheral contacts on the perimeter. Current is injected at contact $j$ and extracted at contact $N$. Contact $N$ is grounded. If the Hall angle is equal to the skew angle, the current density is homogeneous and parallel to the left and right edges, and the electric field is also homogeneous and vertical. Figure 2 is obtained by conformal transformation of Figure 1 . The points $D_{n}$ are the tips of the $N$-2 folded output contacts. They correspond to the stagnation points $d_{n}$ in the $z$-plane of Figure 1 . The sign of the skew angle is important: according to (2a) the vector $\boldsymbol{S}$ is obtained by counter-clockwise rotation of $\boldsymbol{E}$ (for $\theta_{H}>0$, i.e., for $B_{\perp}$ pointing out of the drawing plane and negative majority charge carriers).

(2a) is the complex notation of the general Ohm's law in vector form

$$
\boldsymbol{E}=\rho \boldsymbol{S}+\rho \mu_{H} \boldsymbol{S} \times \boldsymbol{B}_{\perp}
$$

In many materials $\rho$ and $\mu_{H}$ do not depend on $B_{\perp}$ as long as $B_{\perp}$ is sufficiently small (see (11.19) in [14]). However, at strong magnetic field and/or for certain doping concentrations $\rho$ and $\mu_{H}$ may well change versus $B_{\perp}$ (see Chapter 1.1c in [14], see also Chapter 3.3 in [15], also [16]).

In (2a), the conjugate complex of $S(w), E(w)$ point in the directions of the vectors $\boldsymbol{S}, \boldsymbol{E}$, respectively. Thus, taking the conjugate of both sides of (2a) we obtain $\boldsymbol{S}$ by counter-clockwise rotation of $\boldsymbol{E}$ (for $\theta_{H}>0$ ). All $N-2$ output contacts must be horizontal and folded in exactly such a way that the outer boundary becomes a smooth parallelogram (without steps in its left and right edges). Only if all folded output contacts are horizontal they are compatible to homogeneous electric and current density fields in the parallelogram. Then the complex potential in the skewed parallelogram is given by (see (29) in [17])

$$
F_{I}(w)=I_{\text {supply }} R_{\text {sheet }} \frac{w}{2 \cos \left(\theta_{H}\right)^{2}}
$$

The complex electric field in the parallelogram is (see left equalities in (25) in [17])

$$
E(w)=E_{u}-i E_{v}=i \frac{\mathrm{d}}{\mathrm{d} w} F_{I}(w)=I_{\text {supply }} R_{\text {sheet }} \frac{i}{2 \cos \left(\theta_{H}\right)^{2}}
$$

and the complex current density in the parallelogram is with (2a)

$$
S(w)=S_{u}-i S_{v}=\frac{1}{2 t_{H}} I_{\text {supply }}\left(i+\tan \left(\theta_{H}\right)\right)
$$


$E(w)$ and $S(w)$ are homogeneous inside the parallelogram-they do not depend on $w$. The current flowing out of the Hall plate via the lower supply contact in Figure 2 becomes

$$
t_{H} \int_{w=-1}^{1}(-1) S_{v} \mathrm{~d} w=I_{\text {supply }}
$$

The supply voltage is given by

$$
V_{\text {supply }}=\int_{\phi=0}^{V_{\text {supply }}} \mathrm{d} \phi=\int_{B_{N}}^{A_{j}} \nabla \phi \cdot \mathrm{d} \boldsymbol{w}=-\int_{v=\operatorname{Im}\left\{B_{N}\right\}}^{\operatorname{Im}\left\{A_{j}\right\}} E_{v} \mathrm{~d} v=I_{\text {supply }} R_{\text {sheet }} \frac{\operatorname{Im}\left\{A_{j}-B_{N}\right\}}{2 \cos \left(\theta_{H}\right)^{2}}
$$

where $\phi$ is the electric potential inside the Hall effect region (the potential on the contacts is denoted by $\left.V_{1}, V_{2}, \cdots, V_{N}\right)$. We are free to choose the ground potential and so we ground the $N$-th contact $\left(V_{N}=0 \Rightarrow \operatorname{Im}\left\{B_{N}=0\right\}\right)$. The current flows out of the Hall plate through the lower contact $N$ of the parallelogram, and it flows into the Hall plate through the upper contact $j$. The electric field in the upper half of the $z$-plane is given by

$$
E(z)=E_{x}-i E_{y}=i \frac{\mathrm{d}}{\mathrm{d} z} F_{I}(z)=i \frac{\mathrm{d} F_{I}(w)}{\mathrm{d} w} \frac{\mathrm{d} w}{\mathrm{~d} z}=I_{\text {supply }} R_{\text {sheet }} \frac{i}{2 \cos \left(\theta_{H}\right)^{2}} \frac{\mathrm{d} w}{\mathrm{~d} z}
$$

whereby we used the fact that due to the conformal mapping between $w$-plane and $z$-plane it holds $F_{I}(z)=F_{I}(w)$. With the Schwartz-Christoffel formula [18] we immediately get the General Formula

$$
\begin{aligned}
& E(z)=I_{\text {supply }} R_{\text {sheet }} \frac{i}{2 \cos \left(\theta_{H}\right)^{2}} K\left(z-b_{1}\right)^{-\left(\frac{\pi / 2+\theta_{H}}{\pi}\right)}\left(z-a_{2}\right)^{-\left(\frac{\pi / 2-\theta_{H}}{\pi}\right)}\left(z-d_{2}\right)^{-\left(\frac{-\pi}{\pi}\right)} \\
& \times\left(z-b_{2}\right)^{-\left(\frac{\pi / 2+\theta_{H}}{\pi}\right)} \cdots\left(z-a_{j}\right)^{-\left(\frac{\pi / 2-\theta_{H}}{\pi}\right)}\left(z-b_{j}\right)^{-\left(\frac{\pi / 2+\theta_{H}}{\pi}\right)}\left(z-a_{j+1}\right)^{-\left(\frac{\pi / 2-\theta_{H}}{\pi}\right)} \\
& \times\left(z-d_{j+1}\right)^{-\left(\frac{-\pi}{\pi}\right)}\left(z-b_{j+1}\right)^{-\left(\frac{\pi / 2+\theta_{H}}{\pi}\right)} \cdots\left(z-a_{N}\right)^{-\left(\frac{\pi / 2-\theta_{H}}{\pi}\right)}\left(z-d_{N}\right)^{-\left(\frac{-\pi}{\pi}\right)} \\
& \times\left(z-b_{N}\right)^{-\left(\frac{\pi / 2+\theta_{H}}{\pi}\right)}\left(z-a_{1}\right)^{-\left(\frac{\pi / 2-\theta_{H}}{\pi}\right)}\left(z-d_{1}\right)^{-\left(\frac{-\pi}{\pi}\right)} \\
& =I_{\text {supply }} R_{\text {sheet }} \frac{i}{2 \cos \left(\theta_{H}\right)^{2}} \frac{K}{L(z)} \prod_{\substack{n=1 \\
n \neq j}}^{N-1}\left(z-d_{n}\right)
\end{aligned}
$$

hereby $K$ is a scaling constant of the Schwartz-Christoffel mapping, contact $N$ is the current drain contact at ground potential, and contact $j$ is the current input contact at supply voltage. All output contacts-i.e., floating contacts with zero current-are folded. Points $D_{n}$ are inner pointed tips of these folded contacts inside the parallelogram in the $w$-plane. They are mapped onto the stagnation points $d_{n}$ on the real axis in the $z$-plane with $a_{n}<d_{n}<b_{n}$ for $n=2,3, \cdots, N-1 ;(n \neq j)$. At contact 1 , it holds $a_{1}<d_{1} \vee b_{1}>d_{1}$. Hence, all $d_{n}$ are real numbers. $K$ is a real number, because for points on the grounded contact $N$ the product $\Pi\left(z-d_{n}\right)$ in the numerator of (8a) is a real number and $L(z)$ is a negative real number (see Table A1 in Appendix A), while the electric field must be perpendicular to contact $N$ with $E_{y}<0$ (the electric field is directed towards the ground node) and therefore $\operatorname{Im}\{E(z)\}>0$. Thus $K>0$ for $a_{1}<d_{1}$ 
and $K<0$ for $d_{1}<b_{1}$.

In the most general case currents are flowing through all contacts. Then the electric field is the following linear superposition:

$$
E(z)=\frac{R_{\text {sheet }} i}{2 \cos \left(\theta_{H}\right)^{2} L(z)} \sum_{j=1}^{N-1} I_{j} K_{j} \prod_{\substack{n=1 \\ n \neq j}}^{N-1}\left(z-d_{n, j}\right)
$$

whereby the real-valued scaling constant $K_{j}$ and the stagnation points $d_{n, j}$ depend on the number $j$ of the contact at which current $I_{j}$ was supplied in Figure 2. Equating the numerators of (1a) and (8b) gives the real constants $c_{n}$ as functions of $d_{n, j}$. This shows that the $c_{n}$ are determined by the stagnation points $d_{n, j}$ of the output contacts. Moreover we see that the highest power in the numerator of (8b) is of order $z^{N-2}$, because the two supply contacts are not folded and therefore they do not contribute to the numerator. Comparison with (1a) gives

$$
c_{N}=0
$$

It means that for $|z| \rightarrow \infty$ the electric field declines with the dominant term $i c_{N-1} z^{-2}$.

The current density in the $z$-plane is given by (2a) if we replace $w=u+i v$ by $z=x+i y$. Integration over the electric field and the current density gives the voltages between the contacts and the currents through the contacts. This was done in [12] with the following results: The real constants $c_{n}$ are linked to the currents $I_{n}$ into the Hall plate through the $n$-th contact

$$
\begin{gathered}
I_{n}=\sum_{k=1}^{N} N_{n, k} c_{k} \\
N_{n, k}=(-1)^{N+n} \frac{\cos \left(\theta_{H}\right)^{2}}{R_{\text {sheet }}} \int_{a_{n}}^{b_{n}} \frac{x^{k-1}}{|L(x)|} \mathrm{d} x \quad \text { for } n=2, \cdots, N \\
N_{1, k}=(-1)^{N+1} \frac{\cos \left(\theta_{H}\right)^{2}}{R_{\text {sheet }}}\left(\int_{-\infty}^{b_{1}} \frac{x^{k-1}}{|L(x)|} \mathrm{d} x+(-1)^{N} \int_{a_{1}}^{\infty} \frac{x^{k-1}}{|L(x)|} \mathrm{d} x\right) \text { for } k<N
\end{gathered}
$$

$$
N_{1, k}=(-1)^{N+1} \frac{\cos \left(\theta_{H}\right)^{2}}{R_{\text {sheet }}}\left(\int_{\max \left(a_{1},-b_{1}\right)}^{\infty} \frac{(-1)^{N} x^{k-1}}{|L(x)|}+\frac{(-x)^{k-1}}{|L(-x)|} \mathrm{d} x+\left\{\begin{array}{l}
\int_{-b_{1}}^{a_{1}} \frac{(-x)^{k-1}}{|L(-x)|} \mathrm{d} x ;-b_{1}<a_{1} \\
-b_{1} \\
\int_{a_{1}} \frac{(-1)^{N} x^{k-1}}{|L(x)|} \mathrm{d} x ; a_{1}<-b_{1}
\end{array}\right) \text { for } k=N\right.
$$

and to the potentials $V_{n}$ at the contacts

$$
\begin{gathered}
U_{n}=V_{n}-V_{n+1}=\sum_{k=1}^{N} M_{n, k} c_{k} \quad \text { for } 1 \leq n \leq N \quad \text { with } V_{N+1}=V_{1} \\
M_{n, k}=(-1)^{N+n} \cos \left(\theta_{H}\right) \int_{b_{n}}^{a_{n+1}} \frac{x^{k-1}}{|L(x)|} \mathrm{d} x
\end{gathered}
$$

Note that $N_{n, k}$ has opposite sign than in [12], because we define $I_{n}>0$ if it flows into the Hall plate through the $n$-th contact. This definition is in accor- 
dance with the general rule for passive $N$-poles in electrical network theory. The sums in (10a), (11a) still comprise the term $c_{N}$ because we want to prove (9) with the following alternative argument: Obviously, the sum of all voltages around the Hall plate must vanish

$$
\begin{aligned}
\sum_{n=1}^{N} U_{n}=0 \Rightarrow & \left(\sum_{n=1}^{N} M_{n, 1}\right) c_{1}+\left(\sum_{n=1}^{N} M_{n, 2}\right) c_{2}+\cdots+\left(\sum_{n=1}^{N} M_{n, N-1}\right) c_{N-1} \\
& +\left(\sum_{n=1}^{N} M_{n, N}\right) c_{N}=0
\end{aligned}
$$

In the appendix we prove with the calculus of residues that the sums in front of $c_{1}, c_{2}, \cdots, c_{N-1}$ vanish, however, the sum in front of $c_{N}$ does not vanish. Therefore (12a) leads again to (9). Moreover, also the sum over all currents must vanish due to Kirchhoffs current law $I_{1}+I_{2}+\cdots+I_{N}=0$.

$$
\begin{aligned}
\sum_{n=1}^{N} I_{n}=0 \Rightarrow & \left(\sum_{n=1}^{N} N_{n, 1}\right) c_{1}+\left(\sum_{n=1}^{N} N_{n, 2}\right) c_{2}+\cdots+\left(\sum_{n=1}^{N} N_{n, N-1}\right) c_{N-1} \\
& +\left(\sum_{n=1}^{N} N_{n, N}\right) c_{N}=0
\end{aligned}
$$

Indeed, in the appendix we can show that the sums in front of all $c_{n}$ with $n<N$ vanish.

\section{The Resistance Matrix of a Hall Plate}

From (12a) we see that we can skip the last equation in (11a) for $n=N$. With (9) it follows that $M_{n, k}$ is a square matrix with $N-1$ columns and rows. With (12b) we can also skip one equation in (10a). Consequently, also $N_{n, k}$ is a square matrix with $N-1$ columns and rows. Since contact $N$ is the grounded supply contact we prefer to skip the last equation in (10a) for $n=N$. This leads to the matrix solution proposed in [19]

$$
\boldsymbol{I}=\left(\begin{array}{c}
I_{1} \\
I_{2} \\
\vdots \\
I_{N-1}
\end{array}\right)=\left(\begin{array}{cccc}
N_{1,1} & N_{1,2} & \cdots & N_{1, N-1} \\
N_{2,1} & N_{2,2} & \cdots & N_{2, N-1} \\
\vdots & \vdots & \ddots & \vdots \\
N_{N-1,1} & N_{N-1,2} & \cdots & N_{N-1, N-1}
\end{array}\right) \cdot\left(\begin{array}{c}
C_{1} \\
C_{2} \\
\vdots \\
C_{N-1}
\end{array}\right)=\boldsymbol{N} \cdot \boldsymbol{c}
$$

and

$$
\boldsymbol{U}=\left(\begin{array}{c}
V_{1}-V_{2} \\
V_{2}-V_{3} \\
\vdots \\
V_{N-1}-V_{N}
\end{array}\right)=\left(\begin{array}{cccc}
M_{1,1} & M_{1,2} & \cdots & M_{1, N-1} \\
M_{2,1} & M_{2,2} & \cdots & M_{2, N-1} \\
\vdots & \vdots & \ddots & \vdots \\
M_{N-1,1} & M_{N-1,2} & \cdots & M_{N-1, N-1}
\end{array}\right) \cdot\left(\begin{array}{c}
c_{1} \\
c_{2} \\
\vdots \\
c_{N-1}
\end{array}\right)=\boldsymbol{M} \cdot \boldsymbol{c}
$$

with the ground node $V_{N}=0$. Eliminating $\boldsymbol{c}$ in (13a) and (13b) gives

$$
\boldsymbol{U}=\boldsymbol{M} \cdot \boldsymbol{N}^{-1} \cdot \boldsymbol{I}
$$

Inserting (B7a) into (14) gives the contact potentials $\boldsymbol{V}=\left(V_{1}, V_{2}, V_{3}, \cdots, V_{N-1}\right)^{\mathrm{T}}$ (the index $T$ denotes the transpose of a vector or a matrix).

$$
\boldsymbol{V}=\boldsymbol{R} \cdot \boldsymbol{I}
$$


In (15a) the resistance matrix $\boldsymbol{R}$ is given by

$$
\begin{aligned}
\boldsymbol{R} & =\Delta^{-1} \cdot \boldsymbol{M} \cdot \boldsymbol{N}^{-1} \\
& =\left(\begin{array}{cccc}
\sum_{\ell=1}^{N-1} M_{\ell, 1} & \sum_{\ell=1}^{N-1} M_{\ell, 2} & \cdots & \sum_{\ell=1}^{N-1} M_{\ell, N-1} \\
\sum_{\ell=2}^{N-1} M_{\ell, 1} & \sum_{\ell=2}^{N-1} M_{\ell, 2} & \cdots & \sum_{\ell=2}^{N-1} M_{\ell, N-1} \\
\vdots & \vdots & \ddots & \vdots \\
M_{N-1,1} & M_{N-1,2} & \cdots & M_{N-1, N-1}
\end{array}\right) \cdot\left(\begin{array}{cccc}
N_{1,1} & N_{1,2} & \cdots & N_{1, N-1} \\
N_{2,1} & N_{2,2} & \cdots & N_{2, N-1} \\
\vdots & \vdots & \ddots & \vdots \\
N_{N-1,1} & N_{N-1,2} & \cdots & N_{N-1, N-1}
\end{array}\right)^{-1}
\end{aligned}
$$

In (15b) the matrix $\Delta$ is defined in Appendix B. The conductance matrix $\boldsymbol{G}$ is given by $\boldsymbol{I}=\boldsymbol{G} \cdot \boldsymbol{V}$. It follows from inversion of (15b). Note that (15b) is computationally not very efficient, because we need to compute two matrices, invert one, and multiply two matrices. Another method is to determine the points $d_{n}$ in (8a) by solving $A_{n}=B_{n}$ on all folded contacts in the parallelogram. This was done in (41) and (42) in [20], but for larger numbers of contacts the higher order algebraic equations for $d_{n}$ apparently cannot be solved in closed form. Conversely, (15b) solves the conduction problem in Hall plates with an arbitrary number of contacts in a straightforward manner-and this is the true achievement of [12] [19].

\section{The Resistance Matrix of the Complementary Hall Plate}

Figure 3 shows a Hall plate which is complementary to the original Hall plate in Figure 1. All contacts and insulating boundaries are swapped. This means

$$
\bar{a}_{n}=b_{n} \text { and } \bar{b}_{n}=a_{n+1} \text { for } n=1,2, \cdots, N
$$

whereby the quantities of the complementary device are denoted by an overbar. Note that in the complementary device all indices rotate by one instance to the right. If the device is mapped onto a circular Hall plate, the indices in the complementary circular device rotate one instance in positive mathematical sense (counterclockwise). If we walk along the boundary in the direction of increasing indices the conductive region is at the left hand side. Therefore, in Figure 3 the complementary Hall plate has an insulating boundary at infinity and the rightmost contact is grounded.

(16) states that in a complementary Hall plate $a_{n}$ and $b_{n}$ are swapped. Comparison with (1b) shows that $L(z)$ remains unchanged if we additionally swap $\gamma \leftrightarrow 1-\gamma$ in the complementary device, which means that the applied magnetic field acting on the complementary Hall plate has opposite polarity. However, in the theory of Homentcovschi and Bercia we see that identical $L(z)$ in the complementary Hall plate would give wrong boundary conditions, because the roles of insulating boundaries and contacts are swapped (see (6) in [12]). Therefore we have to construct a new $L(z)$ for the complementary Hall plate and repeat the calculation of [12]. We use

$$
\bar{L}(z)=\exp (-i \pi \bar{\gamma}) \prod_{n=1}^{N}\left(z-\bar{a}_{n}\right)^{1-\bar{\gamma}}\left(z-\bar{b}_{n}\right)^{\bar{\gamma}}
$$




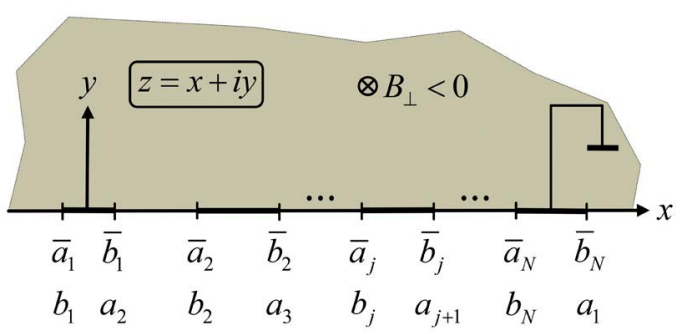

Figure 3. Hall plate in the infinite upper half of the $z$-plane. The device is complementary to the Hall plate in Figure 1 and it is exposed to reverse magnetic field (denoted by $\otimes$ ). The complementary Hall plate has also $N$ peripheral contacts on the real axis. Contact $N$ is grounded. An insulating boundary is at infinity.

where $\bar{\gamma}$ denotes the applied magnetic field on the complementary Hall plate

$$
\bar{\gamma}=\frac{1}{2}+\frac{\overline{\theta_{H}}}{\pi} \text { with } 0<\bar{\gamma}<1 \text { and } \tan \overline{\theta_{H}}=\overline{\mu_{H} B_{\perp}}
$$

Now the new product $\bar{L}(z) \bar{E}(z)$ has to fulfil the homogeneous boundary conditions $\operatorname{Re}\{\bar{L}(z) \bar{E}(z)\}=0$ on the entire real axis. Thereby $\bar{E}(z)$ is the electric field in the complementary Hall plate. Following the same arguments as in [12] we get

$$
\bar{E}(z)=\bar{E}_{x}-i \bar{E}_{y}=\frac{i}{\bar{L}(z)} \sum_{n=1}^{N-1} \bar{c}_{n} z^{n-1} \text { with } \bar{c}_{n} \in \mathrm{R}
$$

If the magnetic field is reversed on the complementary Hall plate $\overline{B_{\perp}}=-B_{\perp}$ it holds $\bar{\gamma}=1-\gamma$ and this gives

$$
\left.\bar{L}(z)\right|_{B_{\perp}}=-\exp (i \pi \gamma) \prod_{n=1}^{N}\left(z-a_{n}\right)^{1-\gamma}\left(z-b_{n}\right)^{\gamma}=\left.L(z)\right|_{B_{\perp}} \times(-\exp (i \pi \gamma))
$$

Comparison of (19a) and (1b) gives the important result

$$
\left.|\bar{L}(z)|_{B_{\perp}}|=| L(z)\right|_{B_{\perp}} \mid
$$

Computing the voltages gives with (11a) and Table A1 in Appendix A

$$
\begin{aligned}
\bar{U}_{n}\left(-B_{\perp}\right) & =\bar{V}_{n}\left(-B_{\perp}\right)-\bar{V}_{n+1}\left(-B_{\perp}\right)=-\int_{\text {contac } n}^{\text {contact } n+1} \mathrm{~d} \bar{\phi}=-\int_{\text {contact } n}^{\text {contact } n+1} \nabla \bar{\phi} \cdot \mathrm{d} \boldsymbol{x} \\
& =\int_{\text {contact } n}^{\text {contact } n+1} \bar{E}_{x} \mathrm{~d} x=\int_{\bar{b}_{n}}^{\bar{a}_{n+1}} \operatorname{Re}\{\bar{E}(z)\} \mathrm{d} x \\
& =\int_{a_{n+1}}^{b_{n+1}} \operatorname{Re}\left\{\frac{i \sum_{k=1}^{N-1} \bar{c}_{k} x^{k-1}}{(-1)^{n} \exp (-i \pi \gamma)|L(x)|}\right\} \mathrm{d} x \\
& =\frac{R_{\text {sheet }}}{\cos \left(\theta_{H}\right)} \sum_{k=1}^{N-1} N_{n+1, k}\left(B_{\perp}\right) \bar{c}_{k} \equiv \sum_{k=1}^{N-1} \bar{M}_{n, k}\left(-B_{\perp}\right) \bar{c}_{k}
\end{aligned}
$$

whereby the rightmost equation in (20) defines $\overline{\boldsymbol{U}}\left(-B_{\perp}\right)=\overline{\boldsymbol{M}}\left(-B_{\perp}\right) \cdot \overline{\boldsymbol{c}}\left(-B_{\perp}\right)$ analogous to $\boldsymbol{U}\left(B_{\perp}\right)=\boldsymbol{M}\left(B_{\perp}\right) \cdot \boldsymbol{c}\left(B_{\perp}\right)$ in (13b). This means

$$
\bar{M}\left(-B_{\perp}\right)=\frac{R_{\text {sheet }}}{\cos \left(\theta_{H}\right)} N_{\uparrow}\left(B_{\perp}\right)=\frac{R_{\text {sheet }}}{\cos \left(\theta_{H}\right)} \mathbf{1}_{\uparrow} \cdot N\left(B_{\perp}\right)
$$




$$
\bar{M}_{n, k}\left(-B_{\perp}\right)=\frac{R_{\text {sheet }}}{\cos \left(\theta_{H}\right)} N_{n+1, k}\left(B_{\perp}\right)
$$

In (21a) we used the shift-up operation defined in Appendix B.

$$
\begin{aligned}
\boldsymbol{N}_{\uparrow}=(N)_{m+1, n}= & \left(\begin{array}{cccc}
N_{2,1} & N_{2,2} & \cdots & N_{2, N-1} \\
N_{3,1} & N_{3,2} & \cdots & N_{3, N-1} \\
\vdots & \vdots & \ddots & \vdots \\
N_{N-1,1} & N_{N-1,2} & \cdots & N_{N-1, N-1} \\
-\sum_{\ell=1}^{N-1} N_{\ell, 1} & -\sum_{\ell=1}^{N-1} N_{\ell, 2} & \cdots & -\sum_{\ell=1}^{N-1} N_{\ell, N-1}
\end{array}\right) \\
& =\left(\begin{array}{cccc}
N_{2,1} & N_{2,2} & \cdots & N_{2, N-1} \\
N_{3,1} & N_{3,2} & \cdots & N_{3, N-1} \\
\vdots & \vdots & \ddots & \vdots \\
N_{N-1,1} & N_{N-1,2} & \cdots & N_{N-1, N-1} \\
N_{N, 1} & N_{N, 2} & \cdots & N_{N, N-1}
\end{array}\right)
\end{aligned}
$$

In $N_{\uparrow}$ all rows of $\boldsymbol{N}$ are shifted up once and the last row in $N_{\uparrow}$ is equal to the negative sum of all rows in $N$. For the right equation in (22) we used (A5b) in Appendix A. We can get $N$ from (21a) with (B10a)

$$
\begin{gathered}
\boldsymbol{N}\left(B_{a, z}\right)=\frac{\cos \left(\theta_{H}\right)}{R_{\text {sheet }}} \overline{\boldsymbol{M}}_{\downarrow}\left(-B_{a, z}\right)=\frac{\cos \left(\theta_{H}\right)}{R_{\text {sheet }}} \mathbf{1}_{\downarrow} \cdot \overline{\boldsymbol{M}}\left(-B_{a, z}\right) \\
\boldsymbol{M}_{\downarrow}=\left(\begin{array}{ccccc}
-\sum_{\ell=1}^{N-1} M_{\ell, 1} & -\sum_{\ell=1}^{N-1} M_{\ell, 2} & \cdots & -\sum_{\ell=1}^{N-1} M_{\ell, N-1} \\
M_{1,1} & M_{1,2} & \cdots & M_{1, N-1} \\
\vdots & \vdots & \ddots & \vdots \\
M_{N-3,1} & M_{N-3,2} & \cdots & M_{N-3, N-1} \\
M_{N-2,1} & M_{N-2,2} & \cdots & M_{N-2, N-1}
\end{array}\right) \\
=\left(\begin{array}{ccccc}
M_{N, 1} & M_{N, 2} & \cdots & M_{N, N-1} \\
M_{1,1} & M_{1,2} & \cdots & M_{1, N-1} \\
\vdots & \vdots & \ddots & \vdots \\
M_{N-3,1} & M_{N-3,2} & \cdots & M_{N-3, N-1} \\
M_{N-2,1} & M_{N-2,2} & \cdots & M_{N-2, N-1}
\end{array}\right)
\end{gathered}
$$

with the definition $M_{0 . \ell}=M_{N . \ell}$. For the right equation in (23b) we used (A5a) in Appendix A. The currents flowing into the complementary Hall plate through the contacts are

$$
\begin{aligned}
\bar{I}_{n}\left(-B_{\perp}\right) & =t_{H} \int_{\bar{a}_{n}}^{\bar{b}_{n}} \bar{S}_{y} \mathrm{~d} x=t_{H} \int_{b_{n}}^{a_{n+1}}(-1) \operatorname{Im}\left\{\frac{\cos \left(\bar{\theta}_{H}\right)}{\rho} \exp \left(-i \bar{\theta}_{H}\right) \bar{E}(z)\right\} \mathrm{d} x \\
& =(-1)^{N+n+1} \frac{\cos \left(\theta_{H}\right)}{R_{\text {sheet }}} \int_{b_{n}}^{a_{n+1}} \operatorname{Im}\left\{i \exp \left(i \theta_{H}\right)\right\} \frac{\sum_{k=1}^{N-1} \bar{c}_{k} x^{k-1}}{|L(x)|} \mathrm{d} x \\
& =\frac{-\cos \left(\theta_{H}\right)}{R_{\text {sheet }}} \sum_{k=1}^{N-1} M_{n, k}\left(B_{\perp}\right) \bar{c}_{k} \equiv \sum_{k=1}^{N-1} \bar{N}_{n, k}\left(-B_{\perp}\right) \bar{c}_{k}
\end{aligned}
$$


here we used (11a). The rightmost equation in (24) defines $\overline{\boldsymbol{I}}\left(-B_{\perp}\right)=\overline{\boldsymbol{N}}\left(-B_{\perp}\right) \cdot \overline{\boldsymbol{c}}\left(-B_{\perp}\right)$ analogous to $\boldsymbol{I}\left(B_{\perp}\right)=\boldsymbol{N}\left(B_{\perp}\right) \cdot \boldsymbol{c}\left(B_{\perp}\right)$ in (13a). This means

$$
\bar{N}_{n, k}\left(-B_{\perp}\right)=\frac{-\cos \left(\theta_{H}\right)}{R_{\text {sheet }}} M_{n, k}\left(B_{\perp}\right) \Leftrightarrow \bar{N}\left(-B_{\perp}\right)=\frac{-\cos \left(\theta_{H}\right)}{R_{\text {sheet }}} \boldsymbol{M}\left(B_{\perp}\right)
$$

for $n=1,2, \cdots, N-1$ (we do not need the case $n=N$ ). (21) and (25) state that all elements of the resistance and conductance matrix of the complementary Hall plate at reverse magnetic field can be derived from the matrices $\boldsymbol{M}$ and $\boldsymbol{N}$ of the original Hall plate at non-reverse magnetic field. We call (21) and (25) the reverse magnetic field on complementary device theorem (RMFoCD).

With the RMFoCD theorem we can predict the behavior of complementary Hall plates from the original ones. Figure 4 shows a circuit where currents are injected into the contacts of a Hall plate with ideal current sources. This is the usual way how Hall plates are operated, because the supply current is forced through the device with a current source while zero current is guaranteed at the output contacts when one connects ideal voltmeters there. In general, it holds with (14)

$$
\boldsymbol{I}\left(B_{\perp}\right)=\boldsymbol{N}\left(B_{\perp}\right) \cdot \boldsymbol{M}^{-1}\left(B_{\perp}\right) \cdot \boldsymbol{U}\left(B_{\perp}\right)
$$

Multiplying (26a) with $\mathbf{1}_{\uparrow}$ from left gives

$$
\mathbf{1}_{\uparrow} \cdot \boldsymbol{I}\left(B_{\perp}\right)=\mathbf{1}_{\uparrow} \cdot \boldsymbol{N}\left(B_{\perp}\right) \cdot \boldsymbol{M}^{-1}\left(B_{\perp}\right) \cdot \boldsymbol{U}\left(B_{\perp}\right)
$$

On the other hand, we can write for the complementary Hall plate at reverse magnetic field with (14)

$$
\bar{U}\left(-B_{\perp}\right)=\bar{M}\left(-B_{\perp}\right) \cdot \overline{\boldsymbol{N}}^{-1}\left(-B_{\perp}\right) \cdot \overline{\mathbf{I}}\left(-B_{\perp}\right)
$$

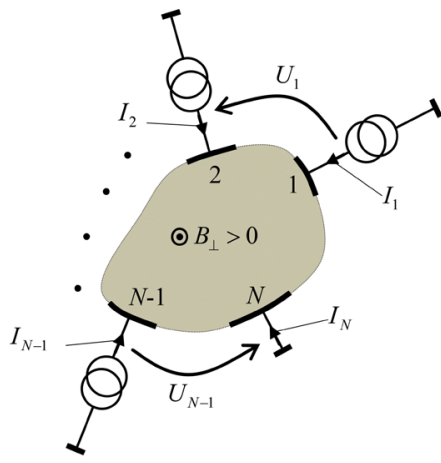

(a)

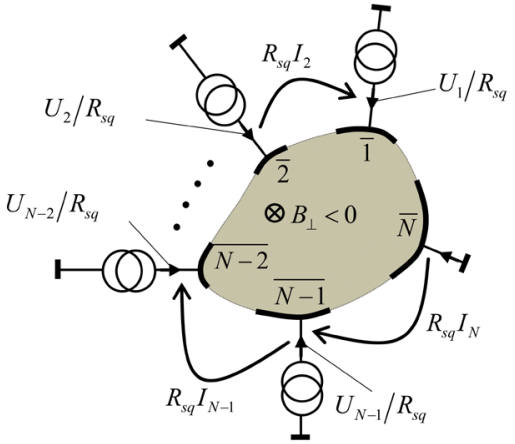

(b)

Figure 4. Reverse magnetic field on complementary device theorem (RMFoCD): The left part (a) shows a Hall plate with $N$ peripheral contacts, where a magnetic field is impressed that points out of the drawing plane (denoted by the circle with the dot inside). The right part (b) shows its complementary Hall plate at reverse magnetic field (denoted by $\otimes$ ). If currents $I_{n}$ are injected into the left Hall plate, voltages $U_{n}$ result. If currents $U_{n} / R_{s q}$ are injected into the complementary device, voltages $-R_{s q} I_{n}$ result. Note that $R_{s q}=R_{\text {sheet }} / \cos \left(\theta_{H}\right)$. 
With (21a) and (25) this is equivalent to

$$
\frac{-\cos \left(\theta_{H}\right)}{R_{\text {sheet }}} \overline{\boldsymbol{U}}\left(-B_{\perp}\right)=\mathbf{1}_{\uparrow} \cdot \boldsymbol{N}\left(B_{\perp}\right) \cdot \boldsymbol{M}^{-1}\left(B_{\perp}\right) \cdot \frac{R_{\text {sheet }}}{\cos \left(\theta_{H}\right)} \overline{\mathbf{I}}\left(-B_{\perp}\right)
$$

(26b) and (27b) are sets of linear equations with identical coefficient matrix $\mathbf{1}_{\uparrow} \cdot \boldsymbol{N}\left(B_{\perp}\right) \cdot \boldsymbol{M}^{-1}\left(B_{\perp}\right)$. Thus, the vectors on the right hand sides must be equal, if the vectors on the left hand sides are equal-and vice versa. This means

$$
\overline{\boldsymbol{U}}\left(-B_{\perp}\right)=-R_{s q} \mathbf{1}_{\uparrow} \cdot \boldsymbol{I}\left(B_{\perp}\right) \Leftrightarrow \overline{\boldsymbol{I}}\left(-B_{\perp}\right)=\frac{1}{R_{s q}} \boldsymbol{U}\left(B_{\perp}\right)
$$

whereby we introduced the square resistance at magnetic field

$$
R_{\text {sq }}=R_{\text {sheet }} \sqrt{1+\mu_{H}^{2} B_{\perp}^{2}}=R_{\text {sheet }} / \cos \left(\theta_{H}\right)
$$

The square resistance is the resistance of a square sample with contacts along two opposite edges (see [21], also (11.36) in [14], also (45) in [22]). Only at zero magnetic field the square resistance is equal to the sheet resistance. If we measure the sheet resistance on a sample with point-sized contacts according to van der Pauw [23], the result does not depend on the applied magnetic field provided $\rho$ is a constant. This can be proven with (16c) in [17], which shows that the tapped voltage in a van der Pauw measurement is constant versus applied magnetic field. Note that (29) differs from the definition of $R_{s q}$ in [19].

Read (28): "if the left equation holds then the right equation follows and vice versa". (28) means that at reverse magnetic field the complementary Hall plate has voltages between neighboring contacts, which are $-R_{s q}$ times the currents in the contacts of the original Hall plate, if the complementary Hall plate is supplied with currents, which are $1 / R_{s q}$ times the voltages between neighboring contacts in the original Hall plate. This is shown in Figure 4.

We can express (28) also in terms of the resistance matrix of the complementary Hall plate at reverse magnetic field. It is defined as

$$
\overline{\boldsymbol{V}}\left(-B_{\perp}\right)=\overline{\boldsymbol{R}}\left(-B_{\perp}\right) \cdot \overline{\boldsymbol{I}}\left(-B_{\perp}\right)
$$

Now we express the left hand side in terms of parameters of the original Hall plate. With (B7c) and the left side of (28) we get

$$
\overline{\boldsymbol{V}}\left(-B_{\perp}\right)=\Delta^{-1} \cdot \overline{\boldsymbol{U}}\left(-B_{\perp}\right)=\frac{-R_{\text {sheet }}}{\cos \left(\theta_{H}\right)} \Delta^{-1} \cdot \mathbf{1}_{\uparrow} \cdot \mathbf{I}\left(B_{\perp}\right)
$$

We also express the current on the right hand side of (30a) in terms of parameters of the original Hall plate. With the right side of (28) and (B7a) we get

$$
\overline{\boldsymbol{I}}\left(-B_{\perp}\right)=\frac{\cos \left(\theta_{H}\right)}{R_{\text {sheet }}} \boldsymbol{U}\left(B_{\perp}\right)=\frac{\cos \left(\theta_{H}\right)}{R_{\text {sheet }}} \Delta \cdot \boldsymbol{V}\left(B_{\perp}\right)
$$

Inserting (30b), (31a) into (30a) and rearranging gives

$$
\boldsymbol{I}\left(B_{\perp}\right)=\frac{-\cos \left(\theta_{H}\right)^{2}}{R_{\text {sheet }}^{2}}\left(\mathbf{1}_{\uparrow}\right)^{-1} \cdot \Delta \cdot \overline{\boldsymbol{R}}\left(-B_{\perp}\right) \cdot \Delta \cdot \boldsymbol{V}\left(B_{\perp}\right)
$$

However, by definition (31b) can also be written as $\boldsymbol{I}\left(B_{\perp}\right)=\boldsymbol{G}\left(B_{\perp}\right) \cdot \boldsymbol{V}\left(B_{\perp}\right)$. 
Comparison with (31b) gives the final result

$$
\begin{gathered}
\overline{\boldsymbol{R}}\left(-B_{\perp}\right)=\frac{-R_{\text {sheet }}^{2}}{\cos \left(\theta_{H}\right)^{2}} \Delta^{-1} \cdot \mathbf{1}_{\uparrow} \cdot \boldsymbol{G}\left(B_{\perp}\right) \cdot \Delta^{-1} \\
\bar{R}_{m, \ell}\left(-B_{\perp}\right)=\frac{-R_{\text {sheet }}^{2}}{\cos \left(\theta_{H}\right)^{2}} \sum_{k=1}^{N-1} \sum_{n=1}^{N-1}\left(\Delta^{-1}\right)_{m, n} G_{n+1, k}\left(B_{\perp}\right)\left(\Delta^{-1}\right)_{k, \ell} \\
=\frac{-R_{\text {sheet }}^{2}}{\cos \left(\theta_{H}\right)^{2}} \sum_{k=1}^{N-1} \sum_{n=m}^{N-1} G_{n+1, k}\left(B_{\perp}\right)\left(\Delta^{-1}\right)_{k, \ell} \\
=\frac{-R_{\text {sheet }}^{2}}{\cos \left(\theta_{H}\right)^{2}} \sum_{k=1}^{\ell} \sum_{n=m}^{N-1} G_{n+1, k}\left(B_{\perp}\right) \\
=\frac{R_{\text {sheet }}^{2}}{\cos \left(\theta_{H}\right)^{2}} \sum_{n=1}^{m} \sum_{k=1}^{\ell} G_{n, k}\left(B_{\perp}\right)
\end{gathered}
$$

In the last transformation we used the identity $\sum_{n=1}^{m} G_{n, k}+\sum_{n=m+1}^{N} G_{n, k}=0$. The sum over all $N$ elements per row or column in the indefinite conductance matrix vanishes [24]. To give an illustrative example: A complementary Hall plate with four contacts at reverse magnetic field has the following resistance matrix

$$
\begin{aligned}
& \overline{\boldsymbol{R}}\left(-B_{\perp}\right)=\frac{R_{\text {sheet }}^{2}}{\cos \left(\theta_{H}\right)^{2}} \\
& \times\left(\begin{array}{ccc}
G_{1,1} & G_{1,1}+G_{1,2} & G_{1,1}+G_{1,2}+G_{1,3} \\
G_{1,1}+G_{2,1} & G_{1,1}+G_{1,2}+G_{2,1}+G_{2,2} & G_{1,1}+G_{1,2}+G_{1,3}+G_{2,1}+G_{2,2}+G_{2,3} \\
G_{1,1}+G_{2,1}+G_{3,1} & G_{1,1}+G_{1,2}+G_{2,1}+G_{2,2}+G_{3,1}+G_{3,2} & G_{1,1}+G_{1,2}+G_{1,3}+G_{2,1}+G_{2,2}+G_{2,3}+G_{3,1}+G_{3,2}+G_{3,3}
\end{array}\right)
\end{aligned}
$$

whereby all $G_{m, n}$ of the original Hall plate are at non-reversed magnetic field $G_{m, n}=G_{m, n}\left(B_{\perp}\right)$. Matrix inversion of both sides of (32a) gives with (B10a)

$$
\overline{\boldsymbol{G}}\left(-B_{\perp}\right)=\frac{-\cos \left(\theta_{H}\right)^{2}}{R_{\text {sheet }}^{2}} \Delta \cdot \boldsymbol{R}\left(B_{\perp}\right) \cdot \mathbf{1}_{\downarrow} \cdot \Delta
$$

On the other hand we can multiply both sides of (32a) first with $\Delta$ and then with $\left(\mathbf{1}_{\uparrow}\right)^{-1}$ from left and with $\Delta$ from right to get

$$
\boldsymbol{G}\left(B_{\perp}\right)=\frac{-\cos \left(\theta_{H}\right)^{2}}{R_{\text {sheet }}^{2}} \mathbf{1}_{\downarrow} \cdot \Delta \cdot \overline{\boldsymbol{R}}\left(-B_{\perp}\right) \cdot \Delta
$$

Matrix inversion of both sides of (34) gives with (B10a)

$$
\boldsymbol{R}\left(B_{\perp}\right)=\frac{-R_{\text {sheet }}^{2}}{\cos \left(\theta_{H}\right)^{2}} \Delta^{-1} \cdot \overline{\boldsymbol{G}}\left(-B_{\perp}\right) \cdot \Delta^{-1} \cdot \mathbf{1}_{\uparrow}
$$

With (B10c) and (B7d) we can write this

$$
\begin{aligned}
R_{m, \ell}\left(B_{\perp}\right) & =\frac{R_{\text {sheet }}^{2}}{\cos \left(\theta_{H}\right)^{2}} \sum_{k=1}^{N-1} \sum_{n=1}^{N-1}\left(\Delta^{-1}\right)_{m, n} \bar{G}_{n, k}\left(-B_{\perp}\right)\left(\Delta^{-1}\right)_{\ell, k} \\
& =\frac{R_{\text {sheet }}^{2}}{\cos \left(\theta_{H}\right)^{2}} \sum_{n=m}^{N-1} \sum_{k=\ell}^{N-1} \bar{G}_{n, k}\left(-B_{\perp}\right)
\end{aligned}
$$




\section{Impedance Relation between Complementary Hall Plates}

In the introduction we have already stated that there is a relation between impedances of original and complementary Hall plates at vanishing magnetic field [1]. With our findings above we can readily give a more general relation at arbitrary magnetic field. Both circuits are sketched in Figure 5. In the original Hall plate we choose the $m$-th contact to inject current. It holds

$$
V_{\text {supply }}=R_{m, m}\left(B_{\perp}\right) I_{\text {supply }}
$$

In the complementary Hall plate we short all contacts left of a line through $m$-th and $N$-th contacts of the original device, and we ground all other contacts. Then we inject current at reverse magnetic field. It holds

$$
\left(\begin{array}{c}
\bar{I}_{1}\left(-B_{\perp}\right) \\
\vdots \\
\bar{I}_{m-1}\left(-B_{\perp}\right) \\
\bar{I}_{m}\left(-B_{\perp}\right) \\
\vdots \\
\bar{I}_{N-1}\left(-B_{\perp}\right)
\end{array}\right)=\overline{\boldsymbol{G}}\left(-B_{\perp}\right) \cdot\left(\begin{array}{c}
0 \\
\vdots \\
0 \\
\bar{V}_{\text {supply }}\left(-B_{\perp}\right) \\
\vdots \\
\bar{V}_{\text {supply }}\left(-B_{\perp}\right)
\end{array}\right)
$$

From which we get

$$
\bar{I}_{\text {supply }}\left(-B_{\perp}\right)=\sum_{n=m}^{N-1} \bar{I}_{n}\left(-B_{\perp}\right)=\sum_{n=m}^{N-1} \sum_{k=m}^{N-1} \bar{G}_{n, k}\left(-B_{\perp}\right) \bar{V}_{\text {supply }}\left(-B_{\perp}\right)
$$

Inserting (35b) into (38) gives

$$
\bar{I}_{\text {supply }}\left(-B_{\perp}\right)=\frac{\cos \left(\theta_{H}\right)^{2}}{R_{\text {sheet }}^{2}} R_{m, m}\left(B_{\perp}\right) \bar{V}_{\text {supply }}\left(-B_{\perp}\right)
$$

Comparison of (39) with (36) gives the result

$$
\frac{\bar{I}_{\text {supply }}\left(-B_{\perp}\right)}{\bar{V}_{\text {supply }}\left(-B_{\perp}\right)}=\frac{\cos \left(\theta_{H}\right)^{2}}{R_{\text {sheet }}^{2}} \frac{V_{\text {supply }}\left(B_{\perp}\right)}{I_{\text {supply }}\left(B_{\perp}\right)}
$$

which we can re-write with (29)
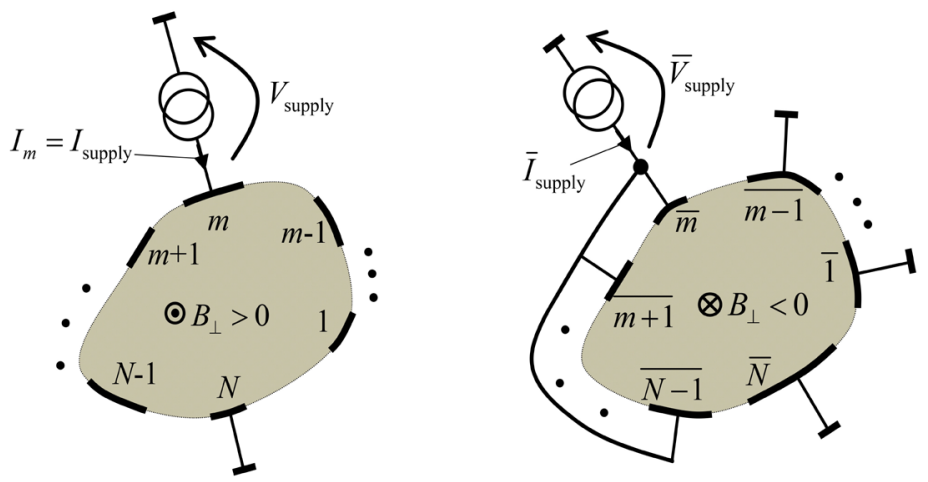

Figure 5. Relation of impedances between complementary Hall plates at opposite magnetic field: The product of resistances $V_{\text {supply }} / I_{\text {supply }}$ and $\bar{V}_{\text {supply }} / \bar{I}_{\text {supply }}$ equals $R_{\text {sheet }}^{2}\left(1+\mu_{H}^{2} B_{\perp}^{2}\right)$. This holds also if the magnetic field is not reversed. 


$$
\left(\frac{1}{R_{\text {sq }}} \frac{V_{\text {supply }}\left(B_{\perp}\right)}{I_{\text {supply }}\left(B_{\perp}\right)}\right) \times\left(\frac{1}{R_{\text {sq }}} \frac{\bar{V}_{\text {supply }}\left(-B_{\perp}\right)}{\bar{I}_{\text {supply }}\left(-B_{\perp}\right)}\right)=1
$$

It means: The product of the numbers of squares of both resistances is equal to 1 . Thereby the number of squares of a resistance is defined as the ratio of this resistance over the square resistance-not over the sheet resistance (see (29)). (40b) also means that the product of both measured resistances is equal to the square of the square resistance:

$$
\frac{V_{\text {supply }}\left(B_{\perp}\right)}{I_{\text {supply }}\left(B_{\perp}\right)} \times \frac{\bar{V}_{\text {supply }}\left(-B_{\perp}\right)}{\bar{I}_{\text {supply }}\left(-B_{\perp}\right)}=R_{s q}^{2}=R_{\text {sheet }}^{2}\left(1+\mu_{H}^{2} B_{\perp}^{2}\right)
$$

This opens up a way to measure the sheet resistance and the Hall mobility of an unknown material. Note that we are free to choose contact $m$ in Figure 5. Hence, for large $N$ we have a large number of possibilities to adjust the impedance level of the circuit to the limitations of our measurement equipment. We only need two Hall plates of identical material with complementary contact geometries. Thereby, reversal of the magnetic field polarity is not necessary here, because the impedance does not depend on the sign of the applied magnetic field. Consistency of measurements with various contact combinations may be used to check for contact quality.

It seems possible to extend this scheme for $R_{m, \ell}\left(B_{\perp}\right)$ with $m \neq \ell$ in (36), but we do not pursue this further.

\section{Complementary Hall Plates with Three Contacts}

Let us consider general Hall plates with three peripheral contacts. Current is injected into contact 1 , voltage is tapped between contact 2 and 3 , whereby contact 3 is the current sink and ground node at zero volts (see Figure 6). In practice, these devices are not the first choice, but sometimes one is obliged to work with them, e.g., for Vertical Hall effect devices [25].

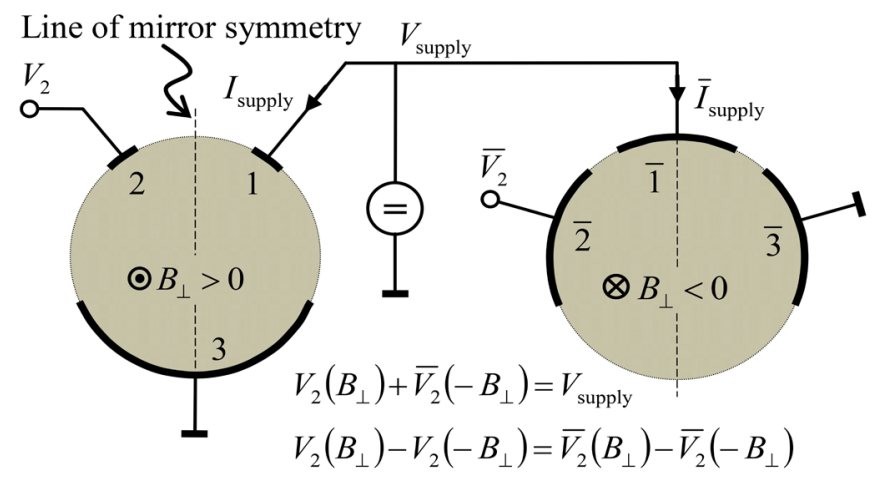

Figure 6. Hall plates with three contacts and a single mirror symmetry: original device at positive magnetic field (left) and complementary device at negative magnetic field (right). Both Hall plates are supplied by the same voltage source. It holds $V_{2}+\bar{V}_{2}=V_{\text {supply }}$ and therefore $V_{2 H}=-\bar{V}_{2 H}$, i.e., the Hall voltages of both Hall plates are equally strong at arbitrary magnetic field. This is a consequence of the RMFoCD theorem. 
If such Hall plates have a single mirror symmetry, and if we supply the original and the complementary Hall plate with the same supply voltage, their output voltages are identical. This was proven for weak magnetic field in [10] [11] with considerable mathematical effort, and with the above theory we will prove it also for strong magnetic field. For the original Hall plate in Figure 6 we write

$$
\left(\begin{array}{c}
I_{\text {supply }} \\
0
\end{array}\right)=\left(\begin{array}{ll}
G_{11} & G_{12} \\
G_{21} & G_{22}
\end{array}\right) \cdot\left(\begin{array}{c}
V_{\text {supply }} \\
V_{2}
\end{array}\right)
$$

which gives the output voltage

$$
V_{2}=\frac{-G_{21}}{G_{22}} V_{\text {supply }}
$$

At zero magnetic field the voltage $V_{2}$ does not vanish-this is called the offset voltage. Therefore $V_{2}$ is of little use in practical sensor applications. However, if one subtracts the voltage $V_{2}$ at positive and negative magnetic field, this procedure cancels out the offset voltage and renders the so-called Hall voltage $V_{2 H}$.

$$
V_{2 H}=\frac{V_{2}\left(B_{\perp}\right)-V_{2}\left(-B_{\perp}\right)}{2}
$$

In other words, the voltage $V_{2}$ may be decomposed into even and odd functions versus $B_{\perp}$ and the odd one is the Hall voltage (see also [2] [17]). With the principle of reverse magnetic field reciprocity (RMFR, see [26]) it holds

$$
G_{m, n}\left(-B_{\perp}\right)=G_{n, m}\left(B_{\perp}\right) \text { for } 1 \leq m, n \leq N-1
$$

Using (43) in (42) we get

$$
V_{2 H}=\frac{G_{12}-G_{21}}{2 G_{22}} V_{\text {supply }}
$$

where the $G_{m, n}$ refer to non-reversed magnetic fields $G_{m, n}=G_{m, n}\left(B_{\perp}\right)$. For the complementary Hall plate in Figure 6 we have

$$
\left(\begin{array}{c}
V_{\text {supply }} \\
\bar{V}_{2}
\end{array}\right)=\left(\begin{array}{cc}
\bar{R}_{11}\left(-B_{\perp}\right) & \bar{R}_{12}\left(-B_{\perp}\right) \\
\bar{R}_{21}\left(-B_{\perp}\right) & \bar{R}_{22}\left(-B_{\perp}\right)
\end{array}\right) \cdot\left(\begin{array}{c}
\bar{I}_{\text {supply }} \\
0
\end{array}\right)
$$

where the quantities with overbar are at reverse magnetic field. It follows

$$
\bar{V}_{2}\left(-B_{\perp}\right)=\frac{\bar{R}_{21}\left(-B_{\perp}\right)}{\bar{R}_{11}\left(-B_{\perp}\right)} V_{\text {supply }}=\frac{G_{11}+G_{21}}{G_{11}} V_{\text {supply }}
$$

For the right equation in (45b) we used (32c). The Hall voltage for the complementary Hall plate is given by

$$
\bar{V}_{2 H}\left(-B_{\perp}\right)=\frac{G_{21}-G_{12}}{2 G_{11}} V_{\text {supply }}
$$

where $\bar{V}_{2 H}$ is the Hall voltage of the complementary device at reverse magnetic field, yet $G_{11}, G_{12}, G_{21}$ are the conductances of the original device at non-reverse magnetic field. Thus, for asymmetric Hall plates neither the voltages nor the Hall 
voltages are identical for the original and for the complementary devices: $V_{2} \neq \bar{V}_{2}$ and $V_{2 H} \neq \bar{V}_{2 H}$. However, if the original Hall plate has a single mirror symmetry with the symmetry line through the grounded reference contact 3 , it holds $G_{1,1}=G_{2,2}$. Then it follows

$$
V_{2}\left(B_{\perp}\right)+\bar{V}_{2}\left(-B_{\perp}\right)=V_{\text {supply }} \text { and } \bar{V}_{2 H}\left(-B_{\perp}\right)=-V_{2 H}\left(B_{\perp}\right) \text { for } G_{1,1}=G_{2,2}
$$

Note that the complementary device also has a single mirror symmetry, but this symmetry line does not go through the grounded reference contact $\overline{3}$. Swapping the roles of original and complementary Hall plate we conclude that (46) holds also if the symmetry line does not go through the grounded reference contact. Thus, our proof is complete: Hall plates with three contacts and single mirror symmetry have identical Hall output voltages as their complementary Hall plates when they are supplied by voltage sources-and this holds for all Hall angles.

\section{Complementary Hall Plates with $90^{\circ}$ Symmetry}

Let us consider Hall plates with four peripheral contacts and two perpendicular mirror symmetries. Moreover, the contacts should be such that the resistance between two non-neighboring contacts equals the resistance between the other two non-neighboring contacts. All devices with $90^{\circ}$ symmetry belong to this group of Hall plates. However, also rectangular shapes with properly chosen contact sizes have such properties. Some popular shapes are shown in Figure 5 of [7]. The two axes of perpendicular mirror symmetry guarantee that the average of the potentials of the output contacts is half of the supply potential, i.e., the common mode output voltage is half the supply voltage. Even if these devices are not $90^{\circ}$ symmetric, they can be mapped with a conformal transformation to devices with $90^{\circ}$ symmetry. Therefore we only need to discuss devices with $90^{\circ}$ symmetry. Then the complementary Hall plate exhibits the same kind of symmetry.

If we supply the original and the complementary Hall plate with the same supply voltage, their output voltages are identical. This was proven for weak magnetic field in [7] with considerable mathematical effort, and with the above theory we will prove it also for strong magnetic field. The circuit is shown in Figure 7. Note that a different amount of current flows through both devices. Thus it is all the more astonishing that the short-circuiting action of the extended contacts in the device with higher resistance is reduced by exactly the same factor as the resistance goes up (the current goes down), so that the output voltage remains identical.

The conductance matrix of a Hall plate with $90^{\circ}$ symmetry has the following symmetry.

$$
\boldsymbol{G}=\left(\begin{array}{ccc}
G_{11} & G_{12} & -G_{11}-G_{12}-G_{21} \\
G_{21} & G_{11} & G_{12} \\
-G_{11}-G_{12}-G_{21} & G_{21} & G_{11}
\end{array}\right)
$$




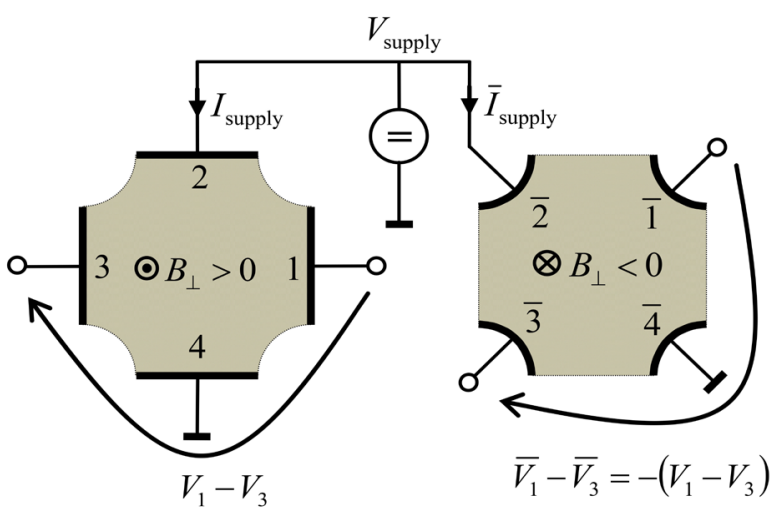

Figure 7. Hall plates with four contacts and $90^{\circ}$ symmetry: at identical magnetic field the original device and the complementary device have identical output voltages, if both Hall plates are supplied by the same voltage source. This is a consequence of the RMFoCD theorem.

This can be proven by applying general currents and voltages to the Hall plate and rotating them by $90^{\circ}$ and $180^{\circ}$. Due to the symmetry of the device the same currents will give the same voltages. This gives 9 equations, which can be solved for the 9 elements of $G$. The solution has the structure of (47). For the original Hall plate in Figure 7 we write

$$
\left(\begin{array}{c}
0 \\
I_{\text {supply }} \\
0
\end{array}\right)=\boldsymbol{G} \cdot\left(\begin{array}{c}
V_{1} \\
V_{\text {supply }} \\
V_{3}
\end{array}\right)
$$

which gives the output voltage

$$
V_{1}-V_{3}=\frac{-G_{12}+G_{21}}{2 G_{11}+G_{12}+G_{21}} V_{\text {supply }}
$$

For the complementary Hall plate in Figure 7 we have

$$
\left(\begin{array}{c}
\bar{V}_{1} \\
V_{\text {supply }} \\
\bar{V}_{3}
\end{array}\right)=\overline{\boldsymbol{R}}\left(-B_{\perp}\right) \cdot\left(\begin{array}{c}
0 \\
\bar{I}_{\text {supply }} \\
0
\end{array}\right)
$$

where the quantities with overbar are at reverse magnetic field. With (32c) the resistance matrix of the complementary device at reverse magnetic field is

$$
\overline{\boldsymbol{R}}\left(-B_{\perp}\right)=\frac{R_{\text {sheet }}^{2}}{\cos \left(\theta_{H}\right)^{2}}\left(\begin{array}{ccc}
G_{1,1} & G_{1,1}+G_{1,2} & -G_{2,1} \\
G_{1,1}+G_{2,1} & 2 G_{1,1}+G_{1,2}+G_{2,1} & G_{1,1}+G_{1,2} \\
-G_{1,2} & G_{1,1}+G_{2,1} & G_{1,1}
\end{array}\right)
$$

which gives the output voltage

$$
\bar{V}_{1}-\bar{V}_{3}=\frac{G_{12}-G_{21}}{2 G_{11}+G_{12}+G_{21}} V_{\text {supply }}
$$

The output voltage of the complementary Hall plate at reverse magnetic field in (49c) has equal magnitude and opposite sign as the output voltage of the original Hall plate at non-reverse magnetic field in (48b), i.e., both devices have 
identical output voltages at identical magnetic field. In general the supply currents are different

$$
\begin{gathered}
I_{\text {supply }}=\frac{2 G_{11}^{2}+G_{12}^{2}+G_{21}^{2}+2 G_{11}\left(G_{12}+G_{21}\right)}{2 G_{11}+G_{12}+G_{21}} V_{\text {supply }} \\
\bar{I}_{\text {supply }}=\frac{1}{2 G_{11}+G_{12}+G_{21}} \frac{\cos \left(\theta_{H}\right)^{2}}{R_{\text {sheet }}^{2}} V_{\text {supply }}
\end{gathered}
$$

If the Hall plates have no symmetry the complementary Hall plate will have different Hall output voltage than the original device when both are supplied by the same voltage source.

Finally we compute the cross-resistances $R_{x}, \bar{R}_{x}$ of original and complementary Hall plates. We define

$$
R_{x}=\frac{V_{1}}{I_{1}+I_{3}} \text { for } V_{1}=V_{3} \text { and } V_{2}=V_{4}=0
$$

From $\boldsymbol{I}=\boldsymbol{G} \cdot \boldsymbol{V}$ it follows with the $90^{\circ}$ symmetry constraints (47)

$$
R_{\chi}=\frac{-1}{2\left(G_{1,2}+G_{2,1}\right)}
$$

From $\overline{\boldsymbol{I}}\left(-B_{\perp}\right)=\overline{\boldsymbol{G}}\left(-B_{\perp}\right) \cdot \overline{\boldsymbol{V}}\left(-B_{\perp}\right)$ it follows with $\overline{\boldsymbol{G}}\left(-B_{\perp}\right)=\overline{\boldsymbol{R}}^{-1}\left(-B_{\perp}\right)$ and (32c) and (47)

$$
\bar{R}_{x}=\frac{-1}{2}\left(G_{1,2}+G_{2,1}\right) \frac{R_{\text {sheet }}^{2}}{\cos \left(\theta_{H}\right)^{2}}
$$

Note that (52) and (53) are independent of the polarity of the magnetic field, because $G_{1,2}\left(B_{\perp}\right)+G_{2,1}\left(B_{\perp}\right)=G_{1,2}\left(B_{\perp}\right)+G_{1,2}\left(-B_{\perp}\right)$ according to the RMFR principle [26]. Comparison of (52) with (53) shows that the square resistance equals twice the square-root of the product of cross-resistances

$$
2 \sqrt{R_{x} \bar{R}_{x}}=R_{\text {sq }}=R_{\text {sheet }} \sqrt{1+\mu_{H}^{2} B_{\perp}^{2}}
$$

(54) is more general than (13a) in [6] because it holds for arbitrary magnetic field. With a measurement of $R_{x}$ and $\bar{R}_{x}$ versus $B_{\perp}$ we can determine $\rho$ and $\left|\mu_{H}\right|$ as functions of $B_{\perp}$ (if $t_{H}$ is known), but we cannot determine the sign of $\mu_{H}$.

\section{Conclusion}

We gave a simple derivation of the electric field in the infinite upper half plane with $N$ contacts on the real axis. The original formula has $N$ real coefficients, but we could show that the $N$-th one vanishes. Based on these results the resistance and conductance matrices of an $N$-contact Hall plate can be expressed in terms of two system matrices $M, N$ with $N$-1 rows and columns. Then we applied this theory to complementary Hall plates where all contacts and insulating boundaries are swapped. It turned out that the resistance matrix of the complementary Hall plate at reverse magnetic field can be expressed by the conductance matrix of the original Hall plate at non-reverse magnetic field. This Reverse 
Magnetic Field on Complementary Device theorem (RMFoCD) was applied to various circuits of Hall plates to prove their symmetry properties. Hall plates with four contacts and $90^{\circ}$ symmetry have identical Hall output voltage as their complementary counterparts, if they are supplied by the same voltage. Hall plates with three contacts and a singly mirror symmetry also have identical Hall output voltage as their complementary counterparts, if they are supplied by the same voltage. For several resistances between contacts or group of contacts in the original and complementary devices, it was shown that their product relates to the square of the square resistance. These findings can be used to determine the resistivity and the magnitude of the Hall mobility of Hall plates with extended contacts as functions of the applied magnetic field. This is an alternative method to van-der-Pauw's method. With due diligence, one might find more relations between Hall plates and their complementary Hall plates with less or no symmetry at all.

\section{Conflicts of Interest}

The author declares no conflicts of interest regarding the publication of this paper.

\section{References}

[1] Homentcovschi, D., Manolescu, A., Manolescu, A.M. and Burileanu, C. (1978) A General Approach to Analysis of Distributed Resistive Structures. IEEE Transactions on Electron Devices, ED-25, 787-794 https://doi.org/10.1109/T-ED.1978.19171

[2] Ausserlechner, U. (2019) The Classical Hall Effect in Multiply-Connected Plane Regions Part II: Spiral Current Streamlines. Journal of Applied Mathematics and Physics, 7, 2231-2264. https://doi.org/10.4236/jamp.2019.710153

[3] Manolescu, A. and Manolescu, A.M. (2007) On Symmetry Properties of Distributed Resistive Structures. 2007 International Symposium on Signals, Circuits and Systems, 1, 1-4. https://doi.org/10.1109/ISSCS.2007.4292675

[4] Cornils, M., Doelle, M. and Paul, O. (2007) Sheet Resistance Determination Using Symmetric Structures with Contacts of Finite Size. IEEE Transactions on Electron Devices, 54, 2756-2761. https://doi.org/10.1109/TED.2007.906219

[5] Ausserlechner, U. (2016) Closed Form Expressions for Sheet Resistance and Mobility from Van-der-Pauw Measurement on $90^{\circ}$ Symmetric Devices with Four Arbitrary Contacts. Solid-State Electronics, 116, 46-55. https://doi.org/10.1016/j.sse.2015.11.030

[6] Ausserlechner, U. (2017) Van-der-Pauw Measurement on Devices with Four Contacts and Two Orthogonal Mirror Symmetries. Solid-State Electronics, 133, 53-63. https://doi.org/10.1016/j.sse.2017.04.006

[7] Ausserlechner, U. (2017) The Signal-to-Noise Ratio and a Hidden Symmetry of Hall Plates. Solid-State Electronics, 135, 14-23. https://doi.org/10.1016/j.sse.2017.06.007

[8] Ausserlechner, U. (2016) A Method to Compute the Hall-Geometry Factor at Weak Magnetic Field in Closed Analytical Form. Electrical Engineering, 98, 189-206. https://doi.org/10.1007/s00202-015-0351-4

[9] Glasser, M.L. and Zhou, Y. (2018) A Functional Identity Involving Elliptic Integrals. 
The Ramanujan Journal, 47, 243-251. https://doi.org/10.1007/s11139-017-9915-4

[10] Ausserlechner, U. (2018) An Analytical Theory of Hall-Effect Devices with Three Contacts. Open Physics Journal, 4, 14-42. https://doi.org/10.2174/1874843001804010014

[11] Ausserlechner, U., Glasser, M.L. and Zhou Y. (2018) Symmetries of Certain Double Integrals Related to Hall Effect Devices. The Ramanujan Journal, 1-8.

[12] Homentcovschi, D. and Bercia, R. (2018) Analytical Solution for the Electric Field in Hall Plates. Zeitschrift für angewandte Mathematik und Physik, 69, 97.

[13] Wick, R.F. (1954) Solution of the Field Problem of the Germanium Gyrator. Journal of Applied Physics, 25, 741-756. https://doi.org/10.1063/1.1721725

[14] Weiss, H. (1969) Structure and Application of Galvanomagnetic Devices. Pergamon Press, Oxford, UK. https://doi.org/10.1016/B978-0-08-012597-8.50009-9

[15] Popovic, R.S. (2004) Hall Effect Devices. 2nd Edition, Institute of Physics Publishing, Bristol, UK.

[16] Marion, D., Cristoloveanu, S. and Chovet, A. (1982) High-Magnetic-Field Van der Pauw Method Magneto Resistance Measurement and Applications. IEE Proceedings I Solid State and Electron Devices, 129, 125-130. https://doi.org/10.1049/ip-i-1.1982.0027

[17] Ausserlechner, U. (2019) The Classical Hall Effect in Multiply-Connected Plane Regions Part I: Topologies with Stream Function. Journal of Applied Mathematics and Physics, 7, 1968-1996. https://doi.org/10.4236/jamp.2019.79136

[18] Betz, A. (1964) Konforme Abbildung. 2nd Edition, Chapter 67, Springer Verlag, Berlin. (In German) https://doi.org/10.1007/978-3-642-87217-4

[19] Homentcovschi, D. and Murray, B.T. (2019) Explicit Resistance Matrix for a Hall Disk with Multiple Peripheral Contacts: Application to a van der Pauw Type Method for Extended Contacts. Sensors and Actuators A: Physical, 294, 1-7. https://doi.org/10.1016/j.sna.2019.04.027

[20] Ausserlechner, U. (2018) An Analytical Theory of the Signal-to-Noise Ratio of Hall Plates with Four Contacts and a Single Mirror Symmetry. Journal of Applied Mathematics and Physics, 6, 2032-2066. https://doi.org/10.4236/jamp.2018.610174

[21] Haeusler, J. (1967) Die Untersuchung von Potentialproblemen bei tensorieller Leitfähigkeit der Halbleiter und Plasmen im transversalen Magnetfeld. Dissertation TH Stuttgart, Chapter 6.1. (In German)

[22] Lippmann, H.J. and Kuhrt, F. (1958) Der Geometrieeinfluß auf den transversalen magnetischen Widerstandseffekt bei rechteckförmigen Halbleiterplatten. Zeitschrift für Naturforschung $A, 13,462-474$. (In German) https://doi.org/10.1515/zna-1958-0608

[23] van der Pauw, L.J. (1958) A Method of Measuring the Resistivity and Hall Coefficient on Lamellae of Arbitrary Shape. Philips Technical Review, 20, 220-224.

[24] Balabanian, N. and Bickart, T.A. (1969) Electrical Network Theory. John Wiley \& Sons, New York.

[25] Ausserlechner, U. (2016) Hall Effect Devices with Three Terminals: Their Magnetic Sensitivity and Offset Cancellation Scheme. Journal of Sensors, 2016, Article ID: 5625607. https://doi.org/10.1155/2016/5625607

[26] Cornils, M. and Paul, O. (2008) Reverse-Magnetic-Field Reciprocity in Conductive Samples with Extended Contacts. Journal of Applied Physics, 104, Article ID: 024505. https://doi.org/10.1063/1.2951895

[27] Boas, R.P. and Boas, H.P. (2010) Invitation to Complex Analysis. 2nd Edition, 
Chapter 11, the Mathematical Association of America, Washington DC.

[28] Carlin, H.J. and Giordano, A.B. (1964) Network Theory: An Introduction to Reciprocal and Nonreciprocal Circuits. Figure 2.17, Prentice-Hall, Upper Saddle River, NJ. 


\section{Appendix A}

From (1b) we get Table A1. With (10b-d) and (11b) this gives

$$
\int_{x=-\infty}^{+\infty} \frac{x^{k-1}}{L(x)} \mathrm{d} x=\frac{-R_{\text {sheet }}}{\cos \left(\theta_{H}\right)^{2}} \sum_{\ell=1}^{N} N_{\ell, k}+\left(\tan \left(\theta_{H}\right)-i\right) \sum_{\ell=1}^{N} M_{\ell, k}
$$

For the integral in (A1) we use the calculus of residues [27]. We integrate along a closed path, which comprises the real axis and a large semi-circle with radius $R \rightarrow \infty$ in the upper half of the $z$-plane. The ends of the contacts represent $2 N$ isolated singular points, which we cut out of the contour integral as shown in Figure A1: we draw a small semi-circle of radius $\varepsilon \rightarrow 0$ around each singular point. The integrals along the small semi-circles vanish as the following example on semi-circle $n$ (denoted by s.c.n) around $a_{n}$ shows

$$
\begin{aligned}
& \lim _{\varepsilon \rightarrow 0} \int_{\text {s.c. } n} \frac{z^{k-1}}{L(z)} \mathrm{d} z \\
& =\lim _{\varepsilon \rightarrow 0} \int_{\vartheta=\pi}^{0} \frac{\varepsilon^{k-1} \exp (i(k-1) \vartheta) \varepsilon \exp (i \vartheta) i \mathrm{~d} \vartheta}{\varepsilon^{1-\gamma} \exp (i(1-\gamma) \vartheta)\left(\prod_{\substack{m=1 \\
m \neq n}}^{N}\left(a_{n}+\varepsilon \exp (i \vartheta)-a_{m}\right)^{1-\gamma}\right)\left(\prod_{m=1}^{N}\left(a_{n}+\varepsilon \exp (i \vartheta)-b_{m}\right)^{\gamma}\right)} \\
& =\lim _{\varepsilon \rightarrow 0} \varepsilon^{k-1+\gamma}=0 \text { for } k=1,2, \cdots, N ; \gamma>0
\end{aligned}
$$

where we used $z=a_{n}+\varepsilon \exp (i \vartheta)$. For the integral along the large semi-circle (denoted by 1.s.c.) we get with $z=R \exp (i \vartheta)$ and for $1 \leq k \leq N$

$$
\begin{aligned}
\lim _{R \rightarrow \infty} \int_{\text {l.s.c. }} \frac{z^{k-1}}{L(z)} \mathrm{d} z & =\lim _{R \rightarrow \infty} \int_{\vartheta=0}^{\pi} \frac{R^{k-1} \exp (i(k-1) \vartheta) R \exp (i \vartheta) i \mathrm{~d} \vartheta}{L(R \exp (i \vartheta))} \\
& =i \int_{\vartheta=0}^{\pi} \lim _{R \rightarrow \infty} R^{k-N} \exp (i(k-N) \vartheta) \mathrm{d} \vartheta=i \pi \delta_{k, N}
\end{aligned}
$$

The integral along the closed contour is proportional to the sum of all enclosed residues. However, this sum is zero, because we cut out all singular points. Therefore we get with (A1), (A2), and (A3)

$$
\begin{aligned}
0 & =\int_{x=-\infty}^{+\infty} \frac{x^{k-1}}{L(x)} \mathrm{d} x+\lim _{R \rightarrow \infty} \int_{\text {l.s.c. }} \frac{z^{k-1}}{L(z)} \mathrm{d} z+\sum_{n=1}^{N} \lim _{\varepsilon \rightarrow 0} \int_{\text {s.c.n }} \frac{z^{k-1}}{L(z)} \mathrm{d} z \\
& =\frac{-R_{\text {sheet }}}{\cos \left(\theta_{H}\right)^{2}} \sum_{\ell=1}^{N} N_{\ell, k}+\left(\tan \left(\theta_{H}\right)-i\right) \sum_{\ell=1}^{N} M_{\ell, k}+i \pi \delta_{k, N}
\end{aligned}
$$

The imaginary part of both sides in (A4) gives

$$
\sum_{\ell=1}^{N} M_{\ell, k}=\pi \delta_{k, N}
$$

with Kronecker's delta $\delta_{k, N}$. With (A5a) the real part of both sides in (A4) gives

$$
\sum_{\ell=1}^{N} N_{\ell, k}=\pi \frac{\sin \left(2 \theta_{H}\right)}{2 R_{\text {sheet }}} \delta_{k, N}
$$

Inserting (A5a) into (12a) and (A5b) into (12b) gives (9). 
Table A1. Phase of the functions $L(x), \bar{L}(x)$ on the real axis (cf. Figure 1 and Figure 3 ).

\begin{tabular}{ccc}
\hline Location of test point $x$ & $L(x) /|L(x)|$ at $B_{\perp}$ & $\bar{L}(x) /|\bar{L}(x)|$ at $-B_{\perp}$ \\
\hline$x<b_{1}$ & $(-1)^{N}$ & $(-1)^{N+1} \exp (i \pi \gamma)$ \\
$b_{1}<x<a_{2}$ & $(-1)^{N} \exp (-i \pi \gamma)$ & $(-1)^{N+1}$ \\
$a_{2}<x<b_{2}$ & $(-1)^{N-1}$ & $(-1)^{N} \exp (i \pi \gamma)$ \\
$b_{2}<x<a_{3}$ & $(-1)^{N-1} \exp (-i \pi \gamma)$ & $(-1)^{N}$ \\
$\vdots$ & $\vdots$ & $\vdots$ \\
$a_{N}<x<b_{N}$ & -1 & $\exp (i \pi \gamma)$ \\
$b_{N}<x<a_{N+1}=a_{1}$ & $-\exp (-i \pi \gamma)$ & 1 \\
$a_{1}<x$ & 1 & $-\exp (i \pi \gamma)$
\end{tabular}

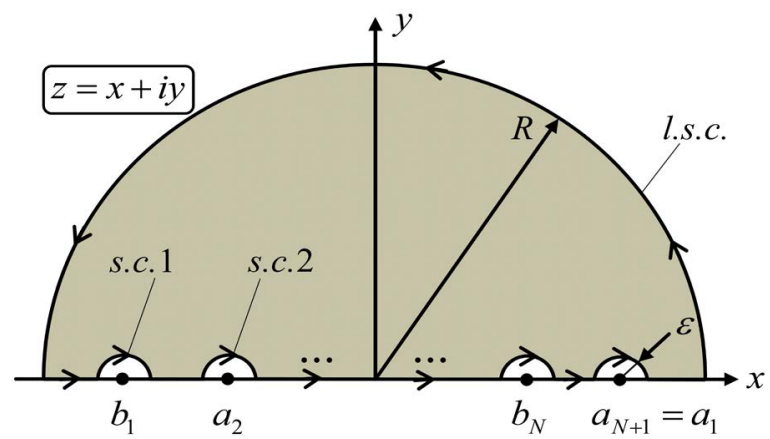

Figure A1. Contour integral along large semi-circle (1.s.c.), real axis, and small semi-circles (s.c.1, s.c.2, ...).

\section{Appendix B}

Here we summarize the mathematics to describe Hall plates with numerous contacts via impedance and conductance matrices. Let us consider a Hall plate with $N$ contacts on the perimeter (see Figure B1). Due to Kirchhoff's nodal current law it holds

$$
\sum_{\ell=1}^{N} I_{\ell}=0
$$

which gives

$$
I_{N}=-\sum_{\ell=1}^{N-1} I_{\ell}
$$

It is common practice to define the potential on one contact as reference potential and to set it equal to zero volts (=ground). We use the potential on the $N$-th contact as reference potential:

$$
V_{N}=0
$$

The voltages between neighboring contacts are defined as

$$
U_{\ell}=V_{\ell}-V_{\ell+1} \text { for } 1 \leq \ell \leq N-1 \text {. }
$$

According to Figure B1 the $N$-th voltage is 


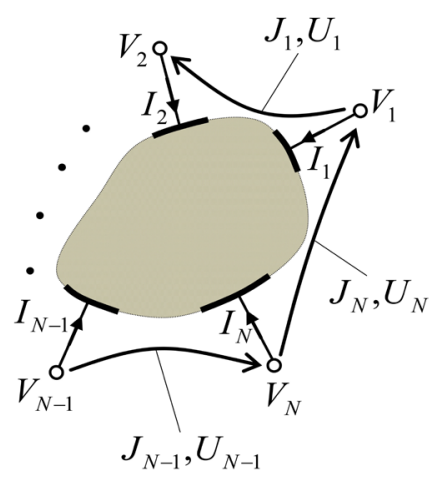

Figure B1. Hall plate with $N$ peripheral contacts. The $N$-th contact is at reference potential (=ground) $V_{N}=0$. Definition of potentials $V_{n}$, currents $I_{n}$, voltages $U_{n}$, and loop currents $J_{n} \cdot J_{N}=0$.

$$
U_{N}=V_{N}-V_{1}=-\sum_{\ell=1}^{N-1} U_{\ell}
$$

which gives

$$
\sum_{\ell=1}^{N} U_{\ell}=0
$$

According to Figure B1 the loop currents $J_{\ell}$ are linked to the currents $I_{\ell}$ via

$$
I_{\ell}=J_{\ell}-J_{\ell-1} \text { for } 2 \leq \ell \leq N
$$

with the first current as

$$
I_{1}=J_{1}-J_{N}
$$

If we add the same current to all loop currents this will not change the currents $I_{\ell}$. Therefore, we are free to choose one loop current. We define

$$
J_{N}=0
$$

With (B1a) it follows

$$
\sum_{\ell=1}^{N} J_{\ell}=0
$$

Thus, the loop current $J_{m}$ flows from terminal $m$ through the Hall plate towards terminal $m+1$, and the voltage $U_{m}$ drops between the same terminals. This definition is in accordance with [28] and it is also shown in Figure B1. To sum up, a Hall plate with $N$ contacts has $N-1$ independent quantities $I_{\ell}, V_{\ell}, U_{\ell}, J_{\ell}$ each. We define vectors with $N-1$ components

$$
\begin{gathered}
\boldsymbol{I}=\left(I_{1}, I_{2}, \cdots, I_{N-1}\right)^{\mathrm{T}} \\
\boldsymbol{V}=\left(V_{1}, V_{2}, \cdots, V_{N-1}\right)^{\mathrm{T}} \\
\boldsymbol{U}=\left(U_{1}, U_{2}, \cdots, U_{N-1}\right)^{\mathrm{T}} \\
\boldsymbol{J}=\left(J_{1}, J_{2}, \cdots, J_{N-1}\right)^{\mathrm{T}}
\end{gathered}
$$

whereby the index $T$ denotes the transpose. Then, we can write (B3a) as a matrix 
formula

$$
\begin{gathered}
\boldsymbol{U}=\boldsymbol{\Delta} \cdot \boldsymbol{V} \text { with } \Delta_{m, n}=\delta_{m, n}-\delta_{m+1, n}, 1 \leq m, n \leq N-1 \\
\Delta \boldsymbol{\Delta}=\left(\begin{array}{ccccccc}
1 & -1 & 0 & \cdots & 0 & 0 \\
0 & 1 & -1 & \cdots & 0 & 0 \\
0 & 0 & 1 & \cdots & 0 & 0 \\
\vdots & \vdots & \vdots & \ddots & \vdots & \vdots \\
0 & 0 & 0 & \cdots & 1 & -1 \\
0 & 0 & 0 & \cdots & 0 & 1
\end{array}\right)
\end{gathered}
$$

$\Delta$ has $N-1$ rows and columns. In (B7a) we used the Kronecker delta $\delta_{m, n}$, which equals 1 if both indices are identical-otherwise it vanishes. The inverse of (B7a) is given by

$$
\begin{gathered}
\boldsymbol{V}=\boldsymbol{\Delta}^{-1} \cdot \boldsymbol{U} \text { with }\left(\Delta^{-1}\right)_{m, n}= \begin{cases}1 & \text { for } n \geq m \\
0 & \text { for } n<m\end{cases} \\
\Delta^{-1}=\left(\begin{array}{cccccc}
1 & 1 & 1 & \cdots & 1 & 1 \\
0 & 1 & 1 & \cdots & 1 & 1 \\
0 & 0 & 1 & \cdots & 1 & 1 \\
\vdots & \vdots & \vdots & \ddots & \vdots & \vdots \\
0 & 0 & 0 & \cdots & 1 & 1 \\
0 & 0 & 0 & \cdots & 0 & 1
\end{array}\right)
\end{gathered}
$$

We define shift-up and shift-down manipulations of vectors:

$$
\boldsymbol{I}_{\uparrow}=\left(\begin{array}{c}
I_{2} \\
I_{3} \\
\vdots \\
I_{N-1} \\
-\sum_{\ell=1}^{N-1} I_{\ell}
\end{array}\right) \text { and } \boldsymbol{I}_{\downarrow}=\left(\begin{array}{c}
-\sum_{\ell=1}^{N-1} I_{\ell} \\
I_{1} \\
\vdots \\
I_{N-3} \\
I_{N-2}
\end{array}\right)
$$

With these manipulations we shift all elements by one place, kick top or bottom elements out, and receive a new element, which is equal to the negative sum of all former elements. Due to (B1b) the new element is equal to $I_{N}$, and the manipulations (B8) do not destroy information. We can apply shift-up and shiftdown to arbitrary vectors. In particular, we can use $\boldsymbol{U}$ instead of $\boldsymbol{I}$. We can shift-up and shift-down also $\boldsymbol{J}$ and $\boldsymbol{V}$ but there the new element $-V_{1}-V_{2}-\cdots-V_{N-1}$ is not equal to $V_{N}$ (the same applies to $\boldsymbol{J}$ ). This is a consequence of our definitions (B2, B5a), which differ from (B1b, B3b). We could also redefine the reference potential $V_{N}$ analogous (B1b) but this would give more complicated matrices in (B7b) and (B7d). We can describe the shift-up procedure by a matrix multiplication:

$$
\boldsymbol{I}_{\uparrow}=\left(\begin{array}{c}
I_{2} \\
I_{3} \\
\vdots \\
I_{N-1} \\
-\sum_{\ell=1}^{N-1} I_{\ell}
\end{array}\right)=\mathbf{1}_{\uparrow} \cdot \boldsymbol{I}=\left(\begin{array}{ccccc}
0 & 1 & 0 & \cdots & 0 \\
0 & 0 & 1 & \cdots & 0 \\
\vdots & \vdots & \vdots & \ddots & \vdots \\
0 & 0 & 0 & \cdots & 1 \\
-1 & -1 & -1 & \cdots & -1
\end{array}\right) \cdot\left(\begin{array}{c}
I_{1} \\
I_{2} \\
\vdots \\
I_{N-2} \\
I_{N-1}
\end{array}\right)
$$


where $\mathbf{1}$ is the $(N-1) \times(N-1)$ identity matrix, and $\mathbf{1}_{\uparrow}$ is obtained from $\mathbf{1}$ by shifting all rows up once and setting the elements of the bottom row equal to the negative sum of all elements per column in the original matrix $\mathbf{1}$. In other words, we apply the shift-up procedure not only to vectors but also to matrices. The shift-down manipulation is also equivalent to a matrix multiplication:

$$
\boldsymbol{I}_{\downarrow}=\left(\begin{array}{c}
-\sum_{\ell=1}^{N-1} I_{\ell} \\
I_{1} \\
\vdots \\
I_{N-3} \\
I_{N-2}
\end{array}\right)=\mathbf{1}_{\downarrow} \cdot \boldsymbol{I}=\left(\begin{array}{cccccc}
-1 & -1 & -1 & \cdots & -1 & -1 \\
1 & 0 & 0 & \cdots & 0 & 0 \\
0 & 1 & 0 & \cdots & 0 & 0 \\
0 & 0 & 1 & \cdots & 0 & 0 \\
\vdots & \vdots & \vdots & \ddots & \vdots & \vdots \\
0 & 0 & 0 & \cdots & 1 & 0
\end{array}\right) \cdot\left(\begin{array}{c}
I_{1} \\
I_{2} \\
\vdots \\
I_{N-2} \\
I_{N-1}
\end{array}\right)
$$

It holds

$$
\left(\mathbf{1}_{\downarrow}\right)^{-1}=\mathbf{1}_{\uparrow}
$$

Therefore, shift-up and shift-down annihilate each other

$$
\mathbf{1}_{\uparrow} \cdot \mathbf{I}_{\downarrow}=\mathbf{1}_{\uparrow} \cdot \mathbf{1}_{\downarrow} \cdot \mathbf{I}=\mathbf{1} \cdot \mathbf{I}=\mathbf{I}
$$

However, we are not allowed to reverse the order: $\left(\mathbf{1}_{\downarrow}\right)^{-1} \neq\left(\mathbf{1}^{-1}\right)_{\downarrow}$. Moreover, it holds

$$
\Delta^{-1} \cdot \mathbf{1}_{\uparrow}=-\left(\Delta^{-1}\right)^{\mathrm{T}}
$$

We can also write (B4a), (B4b) with (B5a) as a matrix relation

$$
\boldsymbol{I}=\Delta^{\mathrm{T}} \cdot \boldsymbol{J}
$$

Throughout this paper we use the following definitions of resistance matrix $\boldsymbol{R}$, impedance matrix $\boldsymbol{Z}$, and conductance matrix $\boldsymbol{G}=\boldsymbol{Y}$ :

$$
\begin{gathered}
\boldsymbol{V}=\boldsymbol{R} \cdot \boldsymbol{I} \\
\boldsymbol{U}=\mathbf{Z} \cdot \boldsymbol{J} \\
\boldsymbol{I}=\boldsymbol{G} \cdot \boldsymbol{V}=\boldsymbol{Y} \cdot \boldsymbol{V}
\end{gathered}
$$

All these matrices have $N-1$ rows and columns, whereby $N$ is the number of contacts of the Hall plate. In [1] the authors used indefinite matrices $\boldsymbol{Y}_{(N \times N)}, \boldsymbol{Z}_{(N \times N)}$, which are defined like in (B12b,c), but which have $N$ rows and columns. Due to (B1a) and (B3C) the indefinite matrices have no inverse, but if we delete the last row and the last column we get the definite matrices $\boldsymbol{Y}, \boldsymbol{Z}$ which can be inverted. From $(B 12 a, c)$ it holds

$$
\boldsymbol{R}^{-1}=\boldsymbol{G}=\boldsymbol{Y}
$$

but $\mathbf{Z}^{-1} \neq \boldsymbol{Y}$ because $\mathbf{Z} \neq \boldsymbol{R}$. The correct relation between $\mathbf{Z}^{-1}$ and $\boldsymbol{Y}$ is obtained by inserting (B7a) and (B11) into (B12b):

$$
\begin{gathered}
\boldsymbol{Z}=\boldsymbol{\Delta} \cdot \boldsymbol{R} \cdot \boldsymbol{\Delta}^{\mathrm{T}} \\
\boldsymbol{Z}^{-1}=\left(\boldsymbol{\Delta}^{\mathrm{T}}\right)^{-1} \cdot \boldsymbol{Y} \cdot \boldsymbol{\Delta}^{-1}
\end{gathered}
$$

For the complementary Hall plate at reverse magnetic field it follows 


$$
\overline{\mathbf{Z}}\left(-B_{\perp}\right)=\Delta \cdot \overline{\boldsymbol{R}}\left(-B_{\perp}\right) \cdot \Delta^{\mathrm{T}}=\frac{R_{\text {sheet }}^{2}}{\cos \left(\theta_{H}\right)^{2}} \mathbf{1}_{\uparrow} \cdot \boldsymbol{G}\left(B_{\perp}\right) \cdot\left(\mathbf{1}_{\uparrow}\right)^{\mathrm{T}}
$$

where we used (32a), (B10c), and (B14a). This can be re-written as

$$
\bar{Z}_{m, n}\left(-B_{\perp}\right)=\frac{R_{\text {sheet }}^{2}}{\cos \left(\theta_{H}\right)^{2}} Y_{m+1, n+1}\left(B_{\perp}\right)
$$

with $Y_{m, n}=G_{m, n}$. At zero magnetic field (B15b) differs slightly from (21) in [1] (in the second indices). There are two reasons for this discrepancy: 1) the contacts of the complementary device are shifted in the direction of lower indices in [1] (whereas they are shifted in the direction of larger indices in this paper), and 2) in Figure 2(a) and Figure 2(b) of [1] it should read $d_{m}$ instead of $d_{m-1}, d_{m+1}$ instead of $d_{m}, d_{\ell}$ instead of $d_{\ell-1}, d_{\ell+1}$ instead of $d_{\ell}$ according to their own definition.

Here is another argument which proves that $\bar{Z}_{m, \ell}\left(-B_{\perp}\right)$ is not proportional to $Y_{m+1, \ell}\left(B_{\perp}\right)$ as claimed for $B_{\perp}=0$ in (21) of [1]: The RMFR principle means $\boldsymbol{R}\left(-B_{\perp}\right)=\boldsymbol{R}^{\mathrm{T}}\left(B_{\perp}\right)$ and $\boldsymbol{Y}\left(-B_{\perp}\right)=\boldsymbol{Y}^{\mathrm{T}}\left(B_{\perp}\right) \quad$ [26]. With (B14a) this gives $\mathbf{Z}\left(-B_{\perp}\right)=\mathbf{Z}^{\mathrm{T}}\left(B_{\perp}\right)$ and therefore $\overline{\mathbf{Z}}\left(-B_{\perp}\right)=\overline{\mathbf{Z}}^{\mathrm{T}}\left(B_{\perp}\right)$. Thus, $\bar{Z}_{m, m}\left(-B_{\perp}\right)=\bar{Z}_{m, m}\left(B_{\perp}\right)$, however, $Y_{m+1, m}\left(-B_{\perp}\right) \neq Y_{m+1, m}\left(B_{\perp}\right)$. In other words, an element on the main diagonal of the $\boldsymbol{Z}$-matrix of the complementary Hall plate cannot be proportional to an off-diagonal element of the $\boldsymbol{Y}$-matrix of the original Hall plate, because this contradicts the RMFR principle (as long as the constant of proportionality is even in $B_{\perp}$ ).

In Appendix $\mathrm{C}$ we check our formulae against finite element simulations to guarantee their correctness. Moreover, we emphasize that all our equations work only if the following rules are applied:

1) The contacts are labeled in ascending order 1 to $N$.

2) If we walk along the perimeter of the Hall plate in the direction of rising contact labels, the conductive region lies at the left hand side.

3) The $N$-th contact is grounded and the $N$-th loop current vanishes.

4) The complementary Hall plate is obtained from the original one, by exchanging all contacts with insulating boundaries and vice versa, whereby the contacts of the complementary device are shifted into the direction of rising contact labels against the respective contacts of the original device (compare Figure 1 with Figure 3).

\section{Appendix C}

Here we give numerical examples, where we compare the results of our theory with finite element simulations (FEM). The FEM was conducted with the $2 \mathrm{D}$ conduction model emdc of COMSOL MULTIPHYSICS with the following conductivity matrix:

$$
\left(\begin{array}{l}
E_{x} \\
E_{y}
\end{array}\right)=\boldsymbol{\rho}\left(\begin{array}{l}
S_{x} \\
S_{y}
\end{array}\right) \text { with } \rho=\rho\left(\begin{array}{cc}
1 & \mu_{H} B_{\perp} \\
-\mu_{H} B_{\perp} & 1
\end{array}\right)
$$


We use realistic values for silicon at room temperature with a phosphorus doping of $2 \times 10^{16} / \mathrm{cm}^{3}: \mu_{H}=0.11 / \mathrm{T}$ and $\rho=0.002924 \Omega \cdot \mathrm{m}$. The thickness of the Hall plates was assumed to be $t_{H}=0.9 \mu \mathrm{m}$. This gives a sheet resistance $R_{\text {sheet }}=3248.89 \Omega$ at zero magnetic field. We assume that $\rho$ and $\mu_{H}$ are constant with $B_{\perp}$. We consider semi-infinite Hall plates according to Figure 1.

The first example has the following parameters: $N=4, a_{1}=10 \sqrt{2} \mu \mathrm{m}$, $a_{2}=-10 \pi \mu \mathrm{m}, a_{3}=-10 \mu \mathrm{m}, a_{4}=10 / 3 \mu \mathrm{m}, b_{1}=-50 \mu \mathrm{m}, \quad b_{2}=-20 \mu \mathrm{m}$, $b_{3}=0 \mu \mathrm{m}, b_{4}=10 / 11 \mu \mathrm{m}$. The Hall angle was $\theta_{H}=\pi / 6=30^{\circ}$, which means a strong magnetic flux density $B_{\perp}=5.24864 \mathrm{~T}$. With (10b), (10c), and (11b) we get the following system matrices

$$
\begin{aligned}
\boldsymbol{N} & =\left(\begin{array}{ccc}
-2.068966952 \times 10^{10} & 14226.81805 & -29.30524564 \\
4.920031176 \times 10^{10} & -1.129662241 \times 10^{6} & 26.53209581 \\
-1.376581519 \times 10^{11} & 389605.9384 & -2.518997254
\end{array}\right) \\
\boldsymbol{M} & =\left(\begin{array}{ccc}
-5.923279984 \times 10^{13} & 2.370993550 \times 10^{9} & -97557.41642 \\
2.715479249 \times 10^{14} & -4.330425950 \times 10^{9} & 72482.27809 \\
-4.514465398 \times 10^{14} & -5.196538114 \times 10^{8} & -1171.994832
\end{array}\right)
\end{aligned}
$$

(A5a) and (A5b) also hold for $k=1,2,3$, and 4. If we inject the currents (in units of amps)

$$
\boldsymbol{I}=\left(\begin{array}{lll}
0.001 & 0 & -0.001 / 7
\end{array}\right)^{\mathrm{T}}
$$

i.e., $I_{1}=1 \mathrm{~mA}, I_{2}=0 \mathrm{~mA}$, and $I_{3}=-1 / 7 \mathrm{~mA}$, we get from (13a)

$$
\boldsymbol{c}=\left(\begin{array}{lll}
-6.868102436 \times 10^{-16} & -8.294336001 \times 10^{-10} & -3.404135684 \times 10^{-5}
\end{array}\right)^{\mathrm{T}}(\mathrm{C}
$$

With (C3b) we may compute the electric field inside the Hall region (Table C1). From (13b) and (B7c) we get (in units of volts)

$$
\begin{gathered}
\boldsymbol{U}=\left(\begin{array}{lll}
1.395086802 & 0.9379037967 & 0.7809727339
\end{array}\right)^{\mathrm{T}} \\
\boldsymbol{V}=\boldsymbol{V}_{\text {theory }}=\left(\begin{array}{lll}
3.113963333 & 1.718876531 & 0.7809727339
\end{array}\right)^{\mathrm{T}}
\end{gathered}
$$

With (15b) we get the resistance matrix (in units of Ohms)

$$
\boldsymbol{R}=\left(\begin{array}{lll}
3443.026651 & 3032.326444 & 2303.443227 \\
2131.222364 & 5315.718944 & 2886.420836 \\
1310.983587 & 1756.075838 & 3710.075970
\end{array}\right)
$$

In the FEM model the upper half of the $z$-plane was modeled as a semi-disk with $40 \mathrm{~cm}$ diameter. The FEM simulation used Lagrange multipliers. The mesh had 10.14 million elements, the equation system had 20.29 million degrees of freedom. The solution time was 2 hours 30 minutes on a desktop computer with Intel Core i7-4930K CPU, ASRock X79 Extreme 6 main-board, 64 GB RAM, and $3.4 \mathrm{GHz}$ clock cycle. The solution of the potentials was (in units of volts)

$$
\boldsymbol{V}_{\text {FEM }}=\left(\begin{array}{lll}
3.11298544 & 1.718219614 & 0.780452059
\end{array}\right)^{\mathrm{T}}
$$

and the difference between (C6a) and (C4b) is 


$$
\boldsymbol{V}_{\text {theory }}-\boldsymbol{V}_{\text {FEM }}=\left(\begin{array}{lll}
0.000977893 & 0.000656917 & 0.000520675
\end{array}\right)^{\mathrm{T}}
$$

The main reason for the difference between the FEM result and the analytical theory seems to come from the finite radius of the semi-disk (Figure C1).

Next we check Figure 4. Therefore, we inject the currents (in units of amperes)

$$
\overline{\boldsymbol{I}}=\cos \left(\theta_{H}\right) \boldsymbol{U} / R_{\text {sheet }}=\left(\begin{array}{lll}
371.789 \times 10^{-6} \quad 249.972 \times 10^{-6} & 208.037 \times 10^{-6}
\end{array}\right)^{\mathrm{T}}(\mathrm{C} 7 \mathrm{a})
$$

into the contacts of the complementary Hall plate, and the sign of the magnetic field was reversed $\bar{\theta}_{H}=-\pi / 6=-30^{\circ}$. With (24) we get the coefficients

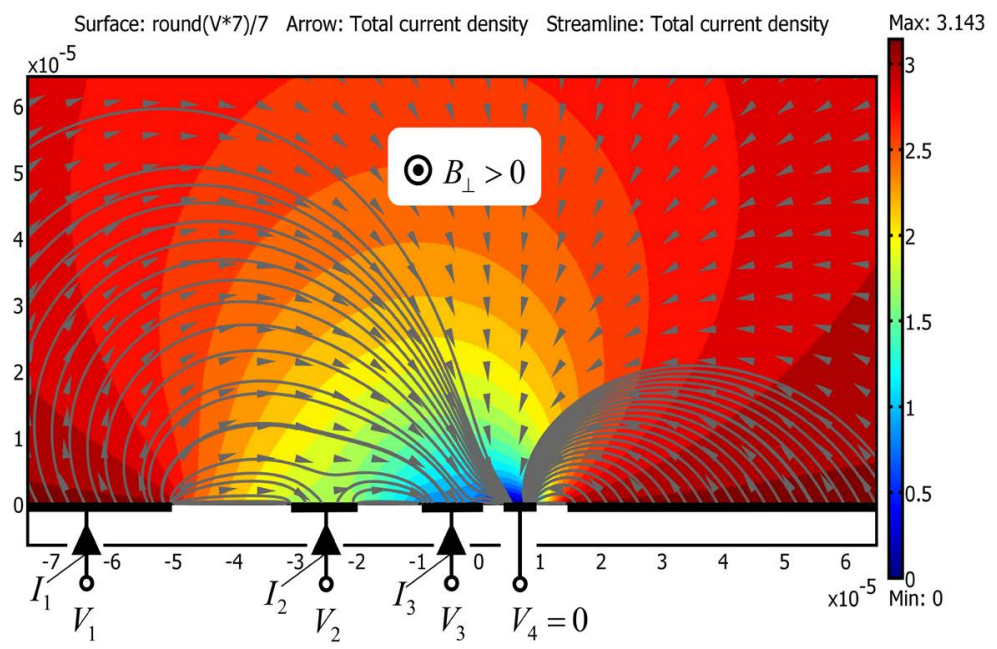

(a)

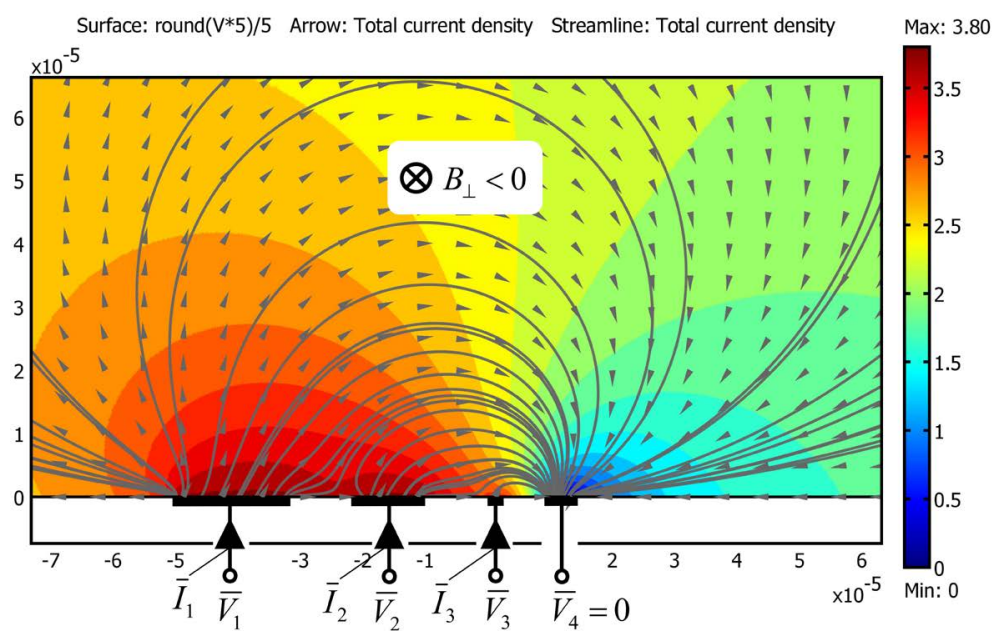

(b)

Figure C1. (a) Hall plate in the infinite upper $z$-plane with four contacts along the real axis at $30^{\circ} \mathrm{Hall}$ angle. (b) Complementary Hall plate at negative magnetic field with $-30^{\circ}$ Hall angle. The figures show the potential according to the color codings given. The current streamlines are grey. The grey cones denote the directions of the current vectors. The contacts are denoted as black, thick, horizontal lines. The scalings on the lower and left edges of the plots denote $x$ - and $y$-grids in units of meters. The currents through the contacts are given in (C3a) and (C7). The potentials at the contacts are given in (C6a) and (C8b). They match with Figure 4. 
Table C1. Electric field vectors on several test points in the original and complementary Hall plate. The table shows numerical values obtained by finite element simulations (FEM) of Figure C1 and by the analytical theory of this work (THEORY). The coordinates of the test points are in $\mu \mathrm{m}$, the electric field is given in $\mathrm{V} / \mathrm{m}$. The electric field vectors of FEM and THEORY match up to $\sim 0.03 \%$ for both Hall plates. Near the edges of the contacts-e.g. in $(x, y)=(3 \mu \mathrm{m}, 0 \mu \mathrm{m})$-the electric field has singularities. There the FEM simulation is inaccurate depending on the mesh.

\begin{tabular}{|c|c|c|c|c|c|c|c|}
\hline Test points $(\mathrm{x}, \mathrm{y})$ : & $(-55,1)$ & $(-55,10)$ & $(-40,3)$ & $(3,0)$ & $(5,7)$ & $(13,0)$ & $(16,13)$ \\
\hline \multicolumn{8}{|c|}{ Original Hall Plate at Positive Magnetic Field in Figure C1(a): } \\
\hline FEM: $E_{\mathrm{x}}$ & 7507.1 & 23185.4 & 41859.8 & 321585.8 & -51865.8 & -297705.8 & -50851.7 \\
\hline FEM: $E_{\mathrm{y}}$ & 48065.3 & 15976.0 & -20193.3 & -185668.8 & -101472.4 & 171882.0 & 1135.6 \\
\hline THEORY: $E_{\mathrm{x}}$ & 7515.7 & 23193.6 & 41851.7 & 321543.9 & -51875.3 & -297592.8 & -50856.6 \\
\hline THEORY: $E_{\mathrm{y}}$ & 48094.4 & 15974.8 & -20191.5 & -185643.4 & -101481.8 & 171815.3 & 1136.3 \\
\hline$\frac{\left|\boldsymbol{E}_{\text {THEORY }}-\boldsymbol{E}_{\text {FEM }}\right|}{\left|\boldsymbol{E}_{\text {THEORY }}\right|}$ & $0.062 \%$ & $0.029 \%$ & $0.018 \%$ & $0.013 \%$ & $0.012 \%$ & $0.038 \%$ & $0.010 \%$ \\
\hline \multicolumn{8}{|c|}{ Complementary Hall Plate at Negative Magnetic Field in Figure C1(b): } \\
\hline Ex (FEM) & -45404.8 & -25425.5 & -3437.9 & 0.0 & 113789.4 & 0.0 & 24436.3 \\
\hline Ey (FEM) & -17535.4 & 12097.9 & 46326.1 & 371068.5 & 5803.1 & -343419.0 & -44599.2 \\
\hline Ex (THEORY) & -45408.8 & -25431.4 & -3439.5 & 0.0 & 113823.5 & 0.0 & $24,444.3$ \\
\hline Ey (THEORY) & -17538.4 & 12098.9 & 46340.4 & 371286.9 & 5815.6 & -343630.6 & $-44,611.3$ \\
\hline$\frac{\left|\boldsymbol{E}_{\text {THEORY }}-\boldsymbol{E}_{\text {FEM }}\right|}{\left|\boldsymbol{E}_{\text {THEORY }}\right|}$ & $0.010 \%$ & $0.021 \%$ & $0.031 \%$ & $0.059 \%$ & $0.032 \%$ & $0.062 \%$ & $0.028 \%$ \\
\hline
\end{tabular}

$$
\overline{\boldsymbol{c}}=\left(\begin{array}{lll}
6.868102436 \times 10^{-16} & 8.294336001 \times 10^{-10} \quad 3.404135684 \times 10^{-5}
\end{array}\right)^{\mathrm{T}}=-\boldsymbol{c}
$$

Inserting (C7b) into (18) gives the electric field in the complementary Hall plate (see Table C1). According to Figure 4 we expect the voltages

$$
\overline{\boldsymbol{U}}_{\text {theory }}=-\left(R_{\text {sheet }} / \cos \left(\theta_{H}\right)\right) \mathbf{1}_{\uparrow} \cdot \mathbf{1}=\left(\begin{array}{lll}
0 & 0.53592768 & 3.21556607
\end{array}\right)^{\mathrm{T}}
$$

The FEM simulation returned the potentials

$$
\overline{\boldsymbol{V}}_{\text {FEM }}=\left(\begin{array}{lll}
3.75095275 & 3.750968675 & 3.214946154
\end{array}\right)^{\mathrm{T}}
$$

from which we get (in volts)

$$
\overline{\boldsymbol{U}}_{\mathrm{FEM}}=\left(\begin{array}{lll}
-0.000015925 & 0.536022521 & 3.214946154
\end{array}\right)^{\mathrm{T}}
$$

The discrepancy is only (in volts)

$$
\overline{\boldsymbol{U}}_{\text {theory }}-\overline{\boldsymbol{U}}_{\mathrm{FEM}}=\left(\begin{array}{lll}
0.000015925 & -0.000094843 & 0.000619917
\end{array}\right)^{\mathrm{T}}
$$

